\title{
The nature of sub-millimetre galaxies II: an ALMA comparison of SMG dust heating mechanisms
}

\author{
B. Ansarinejad ${ }^{1,2 \star}$, T. Shanks ${ }^{1} \dagger$, R.M. Bielby ${ }^{1,3}$, N. Metcalfe ${ }^{1}$, L. Infante ${ }^{4,5,6}$, D.N.A. Murphy ${ }^{7}$ \\ D.J. Rosario ${ }^{1} \&$ S.M. Stach ${ }^{1}$ \\ ${ }^{1}$ Centre for Extragalactic Astronomy, Department of Physics, Durham University, South Road, Durham DH1 3LE, UK \\ ${ }^{2}$ School of Physics, University of Melbourne, Parkville, VIC 3010, Australia \\ ${ }^{3}$ Data Insights and Statistics Division, Department for Education, Bishopsgate House, Darlington, DL1 5QE, UK \\ ${ }^{4}$ Las Campanas Observatory, Carnegie Institution for Science, Colina El Pino S/N, La Serena, Chile \\ ${ }^{5}$ Nucleo de Astronomía de la Facultad de Ingeniería y Ciencias, Universidad Diego Portales, Av. Ejercito Libertador 441, Santiago, 8320000, Chile \\ ${ }^{6}$ Instituto de Astrofisica, Facultad de Fisica, Pontificia Universidad Catolica de Chile, Santiago, Chile \\ ${ }^{7}$ Institute of Astronomy, Madingley Road, Cambridge CB3 OHA, UK
}

Accepted 2021 December 28. Received 2021 December 17; in original form 2021 July 1.

\begin{abstract}
We compare the contribution of Active Galactic Nuclei (AGN) and star-formation towards dust heating in sub-mm galaxies (SMGs). We have used ALMA at 0."1 resolution to image a complete flux-limited sample of seven sub-mm sources previously shown to have spectral energy distributions (SEDs) that were as well-fitted by obscured AGN as star-forming galaxy templates. Indeed, two sub-mm sources were known to be quasars from their absorbed X-ray emission. We find the sub-mm sizes of all SMGs to be small $(\approx 1-2 \mathrm{kpc})$ and generally $\sim 3$ times smaller than any host detected in the Near-Infra-Red (NIR). In all cases, the five SMGs are comparable in sub-mm size to the two known quasars and four $z \approx 6$ quasars, also observed with ALMA. We detect no evidence of diffuse spiral arms in this complete sample. We then convert the Far-Infra-Red (FIR) luminosities to star-formation rate (SFR) surface densities and find that the SMGs occupy the same range as the known quasars in our sample. We conclude that in terms of sub-mm size, extent relative to host and SFR density as well as luminosity and Mid-IR (MIR) colour, there is little distinction between the SMGs and sub-mm bright quasars. Finally, we present preliminary evidence that SMGs with higher MIR luminosities and sub-mm loud quasars tend to have dust components that range to hotter temperatures than their less luminous SMG counterparts. In light of these results, we continue to suggest that luminous SMGs may host dust-absorbed quasars that may simultaneously dominate the FIR and hard X-ray backgrounds.
\end{abstract}

Key words: submillimetre: galaxies - (galaxies:) quasars: supermassive black holes - galaxies: starburst

\section{INTRODUCTION}

Sub-millimetre galaxies (SMGs) were first detected as highly luminous FIR sources (see, e.g. Smail et al. 1997; Barger et al. 1998; Hughes et al. 1998; Dey et al. 1999) using the James Clerk Maxwell Telescope's (JCMT) Submillimetre Common-User Bolometer Array (SCUBA; Holland et al. 1999). These objects were soon found to be high-redshift, dust-obscured sources which contribute to a significant fraction $(\approx 50 \%)$ of the energy output of all galaxies in the early Universe (Blain et al. 2002). Sub-millimetre observations, therefore, opened a new window for studies of galaxy formation and evolution since the cosmic dawn (see Casey et al. 2014 for a detailed review).

The identification of the dominant fuelling mechanism which powers SMGs is an ongoing topic of research. The standard view is that SMGs are predominantly luminous $\left(L_{\mathrm{IR}}>10^{11} \mathrm{~L}_{\odot}\right)$ starburst galaxies (e.g. Sanders \& Mirabel 1996), seen during an obscured phase of their evolution (see, e.g. Sanders \& Mirabel 1996; Alexander

\footnotetext{
^ E-mail:behzad.ansarinejad@unimelb.edu.au (BA)
}

$\dagger$ E-mail: tom.shanks@durham.ac.uk (TS) et al. 2005; Dudzevičiūte et al. 2020). However, it was also found that their high apparent star formation rates (SFR) implied such high stellar masses at such an early epoch, $z \approx 2$, that they presented a problem for the "bottom-up" $\Lambda$-Cold Dark Matter $(\Lambda \mathrm{CDM})$ model. Thus the Semi-Analytic Model of Baugh et al. (2005) significantly under-predicted the abundance of SMGs, requiring the somewhat adhoc adoption of a top-heavy stellar initial mass function in starbursts to increase their luminosity compared to their mass and thus account for the high observed SMG number counts without contradicting the $\Lambda \mathrm{CDM}$ mass function (see also e.g. Geach et al. 2017; Cowley et al. 2019).

An alternative view is that Active Galactic Nuclei (AGN) may be the dominant mechanism that powers luminous SMGs (e.g. Hill \& Shanks 2011a). Although 10-20\% of SMGs are accepted to host AGN (Cowie et al. 2018; Franco et al. 2018; Stach et al. 2019), they are usually viewed to be sub-dominant to star-formation in heating these sources (see, e.g. Laird et al. 2010; Johnson et al. 2013; Wang et al. 2013).

On the other hand, there are various arguments for considering 
obscured AGN as the primary power source for at least, bright, $S_{870 \mu \mathrm{m}} \geq 1 \mathrm{mJy}, \mathrm{SMGs}$. For instance, a population of heavily obscured quasars could explain the missing hard X-ray background (Comastri et al. 1995; Worsley et al. 2005; Polletta et al. 2007), while their dust-rich nature would mean that they would have large emissions in the Infra-Red (IR) due to the emission of reprocessed light from the AGN. Indeed, obscured AGN models have been shown to provide a reasonable fit to the bright end of the SMG source counts (Hill \& Shanks 2011a), reducing the need for a top-heavy IMF for high-redshift starbursts, although issues about the origin of the dust in the AGN may still remain (C.G. Lacey, priv. comm.). Note also that, at fainter flux densities, star-forming galaxies are still expected to dominate the observed SMG number counts. Finally, for AGN to be the dominant source of powering the sub-mm emission rather than star formation, the dust torus must lie far enough (at $\approx$ kiloparsec scales) from the nucleus to maintain a cool temperature of $\approx 35 \mathrm{~K}$ and produce spectra consistent with observations; a picture which is feasible if one assumes a torus model similar to e.g. Kuraszkiewicz et al. (2003).

In recent years, the unprecedented sensitivity and angular resolution of Atacama Large Millimetre/Submillimetre Telescope (ALMA) has enabled the study of the dust heating mechanisms of SMGs at significant redshifts. Using 0." 3 imaging from ALMA, Simpson et al. (2015) found that most of their targeted $z \approx 2$ SMGs are just resolved, with their imaging probing scales of $\approx 2-3 \mathrm{kpc}$. Furthermore, they found the $K$-band optical extent of these SMGs to be roughly four times larger than their extent in sub-mm. Using higher resolution (0."16) ALMA imaging, Hodge et al. (2016) found sub-mm sizes of $\approx 1.3 \mathrm{kpc}$ for $16, S_{870 \mu \mathrm{m}} \approx 3-9 \mathrm{mJy}$, SMGs, with a selection skewed toward the most luminous of the 122 sources in the 'ALMA followup of the LABOCA ECDFS sub-mm survey' (ALESS). Similarly, Gullberg et al. (2019) studied a stacked sample of $\sim 150$ SMGs with 0."18 ALMA resolution, finding a compact sub-mm dust continuum emission extended to just $\sim 1 \mathrm{kpc}$, in comparison to the Hubble Space Telescope (HST) imaging of the optical/UV emissions of the same sources extending to $\sim 8-10 \mathrm{kpc}$. These results may give some rough impression that the better the resolution, the smaller the sub-mm extent that is measured. All studies appear to agree that the sub-mm extent is significantly smaller than the galaxy host in the rest optical, although not all have the benefit of HST imaging.

Then higher resolution (0."08) and, perhaps more importantly, higher S/N ALMA observations by Hodge et al. (2019) found evidence for small-scale (i.e 1-2 kpc radius) spiral arms in a randomly selected (with respect to morphology) sub-sample of 6 of the above 16 luminous ALESS SMGs. More precisely, these 6 SMGs were selected as the sub-mm-brightest sources from the 16 ALESS SMGs with previous high-resolution (0."16) $870 \mu \mathrm{m}$ ALMA imaging from Hodge et al. (2016), which were themselves chosen as the sub-mmbrightest sources with (randomly targeted) HST coverage. These authors attributed the compact size of the sub-mm emitting region of these sources to a central starburst evolving into a galactic bulge or bar. However, an alternative interpretation could be the presence of AGN which are heating the inner regions of these SMGs, producing luminosities comparable to quasar emissions and it is this AGN hypothesis we aim to test further here.

In this paper, we analyse a complete flux-limited sample of 7 sub-mm sources including 5 unidentified $z \approx 2$ SMGs and $2 X$ ray absorbed quasars located in the William Herschel Deep Field (WHDF, e.g. Metcalfe et al. 2006). These sub-mm sources were originally detected by APEX LABOCA (Bielby et al. 2012) and then targetted by ALMA. We also include a sixth fainter SMG, detected by ALMA near one of the quasars. We further include $4 z>6$ quasars originally identified in the VST ATLAS survey (Shanks et al. 2015; Carnall et al. 2015; Chehade et al. 2018) that ALMA has also detected as sub-mm sources.

The WHDF sample is well-suited for comparing AGN and starforming heat sources since it has the combination of high resolution to measure the size of nuclear features and long 1500s exposures to maximise the chance of detecting low surface brightness features such as spiral arms for each target. It also includes two sources that are already known to be QSOs from their X-ray emission and optical spectra that can act as AGN templates. The sample of 5 sources is also complete in the central WHDF area to a fixed flux density limit. The $4 z>6$ quasars act as further high luminosity QSO templates with similar kpc scale resolution and similarly long ALMA exposures.

In the case of the WHDF sub-mm sources, Shanks et al. (2021) (hereafter Paper I) have already compared AGN and star-forming fits to the spectral energy distributions (SEDs) of these sources, constructed using multi-wavelength data ranging from X-ray to radio bands. There, it was found that AGN SEDs fitted most SMGs as well as star-forming galaxy templates and the SMG MIR colours were generally indistinguishable from quasars. Here, we present further comparisons between the SMGs and the quasars of their FIR luminosities, sizes and host galaxy relative extents based on our ALMA FIR continuum imaging. The high ALMA resolution and $\mathrm{S} / \mathrm{N}$ is again competitive with the best previous studies and is clearly advantageous for measuring these properties and making these comparisons.

Measuring the star formation rate surface density, $\sum_{\mathrm{SFR}}$, of SMGs is an additional method of comparing our SMG and quasar subsamples. $\sum_{\text {SFR }}$ can also test whether the observed SFR surface density anywhere exceeds the 'Eddington limit' of star formation set by radiation pressure on $\mathrm{dust}^{1}\left(\sum_{\mathrm{SFR}} \approx 650 \mathrm{M}_{\odot} \mathrm{yr}^{-1} \mathrm{kpc}^{-2}\right.$; see, e.g. Thompson et al. 2005; Walter et al. 2009; Hopkins et al. 2010; Decarli et al. 2018; Hodge et al. 2019), perhaps ultimately requiring an alternative heating mechanism such as AGN.

The outline of this paper is as follows; in Section 2 we describe the observations of our WHDF SMGs, X-ray quasars and $z>6$ quasars. In Section 3, we describe our measurements of the sizes and fluxes of our ALMA sources as well as their host galaxies. In Section 4 we derive FIR luminosities and estimate SFR surface densities based on continuum fluxes and extents. In Section 5 we summarise our comparison of the FIR properties of SMGs and quasars before presenting our conclusions in Section 6. Throughout, we adopt a fiducial cosmology with $H_{0}=70 \mathrm{~km} \mathrm{~s}^{-1} \mathrm{Mpc}^{-1}, \Omega_{\mathrm{m}}=0.3, \Omega_{\Lambda}=0.7$. Finally, note that for calculating SFR's we assume a Chabrier IMF.

\section{OBSERVATIONS}

\subsection{Initial FIR detection of WHDF SMGs and quasars}

Initially, 11 sub-mm sources were detected in an APEX LABOCA (Siringo et al. 2009) $S_{870 \mu \mathrm{m}}>3.3 \mathrm{mJy}$ survey (Bielby et al. 2012). The seven sources subsequently targeted by ALMA, together with an eighth, LAB-06, form a complete flux density limited sample located in the central $7^{\prime} \times 7^{\prime}$ area of the WHDF. LAB-06 was not targeted by ALMA because it is was already identified with a low redshift $(z=0.046)$ spiral galaxy. Two of these sources LAB-05 and LAB-11 have been identified as X-ray absorbed quasars by Bielby et al. (2012)

1 The Eddington limit on $\sum_{\mathrm{SFR}}$ arises due to the momentum deposited due to radiation pressure from stars blowing the star-forming gas and dust out of the system. The value quoted here is a lower bound as estimated by Hodge et al. (2019). 
with redshifts of $z=2.12$ and $z=1.32$ respectively. A summary of the redshifts and coordinates of these sources can be found in Table 1. The redshifts for LAB-05 and LAB-11 are spectroscopic, the other 5 are from the AGN SED fits of Paper I; these have considerably larger errors, typically $\approx 12 \%$. However, the well known dependence of angular diameter distance on redshift means that any redshift with $0.4<z<6.3$ must have a scale within the range $0.54-0.85 \mathrm{kpc} /$ $0 . " 1$. So for a $0 . " 1$ angular scale, the physical scale peaks at $z \approx 1.6$ at $0.85 \mathrm{kpc}$ while a galaxy at $z=6.3$ is as well resolved as a galaxy at $z=0.4$ with a physical scale of $0.54 \mathrm{kpc}$.

\subsection{Initial detection of $z>6$ ATLAS quasars}

The four $z>6$ quasars studied here were initially identified as quasar candidates in the VST ATLAS survey (Shanks et al. 2015) using the colour selection criteria of Carnall et al. (2015) and Chehade et al. (2018). These candidates were confirmed as $z \approx 6$ quasars using lowresolution spectroscopy on the LDSS-3 instrument on the Magellan $6.5 \mathrm{~m}$ telescope, and then with moderate resolution X-Shooter (Vernet et al. 2011) spectra by Chehade et al. (2018). A summary of the properties of these quasars including their black hole masses inferred from the $[\mathrm{CIV}]$ broad emission-line width $M_{\mathrm{BH}}\left[\mathrm{C}_{\mathrm{IV}}\right]$ reported by Chehade et al. (2018) is presented in Table 1. These luminous quasars will be of interest here mainly for comparison with the WHDF SMGs and lower redshift quasars.

\subsection{ALMA observations of the WHDF LABOCA sources}

The flux-limited subset of seven LABOCA sources in the central $7^{\prime} \times$ 7 ' of the WHDF were targeted with ALMA in Band 7 (275-373GHz) on 11/10/2016 with the 12-m Array in a configuration which yielded $870 \mu \mathrm{m}$ continuum images at $0 .{ }^{\prime \prime} 095$ resolution and a maximum recovered scale of $0 . " 926$. The median precipitable water vapour (PWV) at zenith was recorded as $0.65 \mathrm{~mm}$. LAB-01, -02, -03, -04, $-05,-06,-10,-11$ formed the complete flux-limited sample but we excluded LAB-06 as an ALMA target on the grounds that it was already identified with a nearby, $z=0.046$, spiral galaxy. The other seven sources thus comprise our flux-limited sample observed by ALMA. The exposure times were 1572s each, aimed at being long enough to detect any diffuse emission (e.g. spiral arms) surrounding the sub-mm core. These observations reached an $870 \mu \mathrm{m}$ surface brightness rms of $60 \mu \mathrm{Jy}$ per $0 .{ }^{\prime \prime} 09 \times 0 .{ }^{\prime \prime} 11$ beam over $\mathrm{a} \approx 17^{\prime \prime}$ diameter field-of-view. This can be compared to diffuse spiral arms of Hodge et al. (2019) having a surface brightness of typically $200 \mu \mathrm{Jy}$ per $0 .{ }^{\prime \prime} 08 \times 0 .{ }^{\prime \prime} 06$ beam or $400 \mu \mathrm{Jy} / \mathrm{beam}$ at our resolution. Thus similar spiral features should be detected by our observations at $\approx 7 \sigma /$ beam, less than the $\approx 9 \sigma /$ beam of Hodge et al. (2019) but at high significance nonetheless.

To calibrate the observational data, we make use of the standard ALMA data reduction and calibration scripts provided with the raw observations. These scripts were ran with the Common Astronomy Software Application (CASA v4.7.2; McMullin et al. 2007) package and we do not perform any additional tapering or cleaning ${ }^{2}$. All seven sources were strongly detected with LAB-11 revealing a companion at $\approx 5^{\prime \prime}$ from the main LAB-11 source that had been unresolved in the LABOCA data, making an eighth ALMA sub-mm source in the central WHDF area, now named LAB-12. Although this source has an $870 \mu \mathrm{m}$ flux density below 3.3mJy as indeed does

\footnotetext{
2 Note that the ALESS images that we show in Fig. 2(c) have the same
} pipeline reduction consistently applied as for the WHDF images in Fig. 2(a).

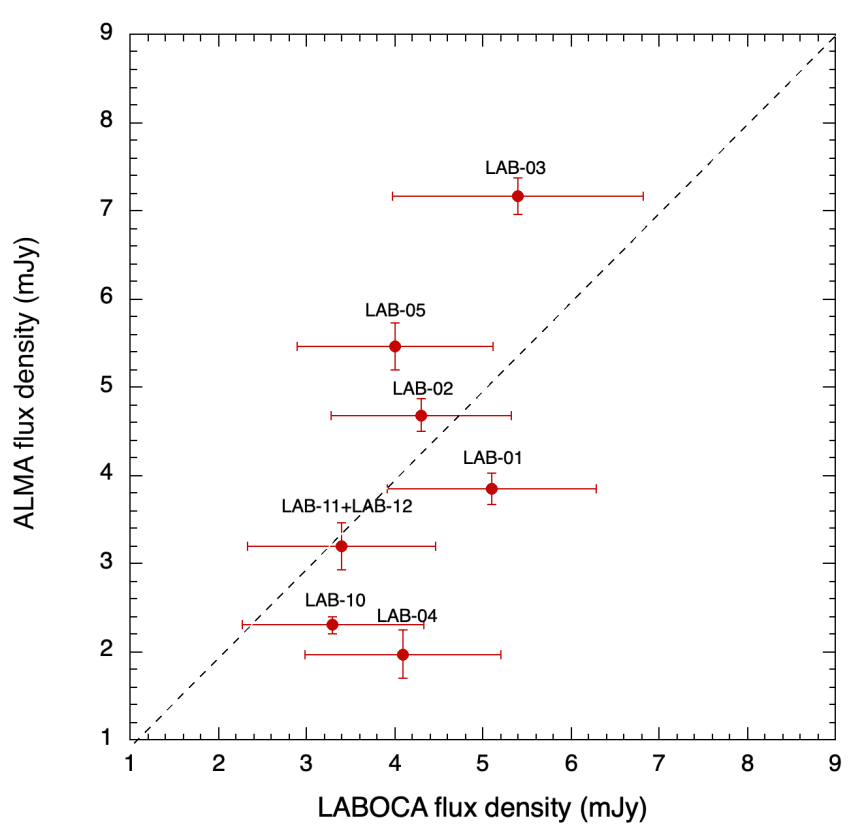

Figure 1. Comparison of ALMA and LABOCA 870 $\mu$ m flux densities for the WHDF SMGs. LAB-11 + LAB-12 flux densities for ALMA are summed in this comparison as they were unresolved in the LABOCA imaging of Bielby et al. (2012).

LAB-11 we shall continue to include these in our main sample, since for our purposes it is more important that our sample is demonstrably unbiased in terms of morphology rather than being specifically flux density limited.

In order to ensure our ALMA observations have recovered the full flux (and therefore the full extent) of the WHDF sources, in Fig. 1 we compare the ALMA flux density of these objects with previous LABOCA flux density measurements of Bielby et al. (2012). Here we have treated LAB-11 and LAB-12 as a single object, summing their ALMA flux densities to find their total ALMA flux density of $3.19 \pm 0.27 \mathrm{mJy}$ before plotting against the LABOCA flux density for LAB-11 of $3.4 \pm 1.06$ mJy. With the possible exception of LAB-04 where the LABOCA flux density appears to be higher than the ALMA flux density (albeit at a $<2 \sigma$ level of significance), the measurements for the remaining sources are in agreement within the error on the LABOCA flux density measurements. In the case of LAB-04, we checked whether any low surface brightness component was missed by trying a range of data smoothing but such tests for LAB-04 and other targets revealed no such components. The lower ALMA flux could be due to the source being placed closer to the edge of the observed ALMA field of view where the ALMA sensitivity and S/N is reduced.

\subsection{ALMA observations of the $z>6$ ATLAS quasars}

The four $z>6$ quasars were targeted with ALMA in Band 6 $(211-275 \mathrm{GHz})$ on $14,17,19$ and $24 / 11 / 2016$ with the 12-m Array in configurations which yielded $\approx 1150 \mu \mathrm{m}$ continuum images at $\approx 0 . .^{\prime \prime} 35-0$. ." 4 resolution and a maximum recovered scale of 3." 4 - 4. " 0 . The exposure times for J029-36, J332-32, J025-33 and J158-14 (SIMBAD IDs: VST-ATLAS J015957.96-363356.8; QSO J2211-3206; QSO J0142-3327; PSO J158.6937-14.4210) were 2956, 1845,1875 and 1996 seconds, reaching continuum sensitivities of $0.020,0.017,0.020$ and $0.014 \mathrm{mJy} / \mathrm{beam}$. Although the spatial res- 
olution of these ALMA observations is $\approx 3-4 \times$ lower than for the WHDF sources, we are able to probe proper distances as small as those at $z \approx 0.5$, due to the reduction of the angular diameter distance, $d_{\mathrm{A}}(z)$, at redshifts $z>1.6$. Taking advantage of this property of the Universe, we were able to resolve $3 / 4$ of our $z \approx 6$ SMGs in the dust continuum.

All four of our $z \approx 6$ quasars were targeted by ALMA for [CII] emission but small uncertainties in the 'discovery' redshifts of two of these sources (J332-23 and J029-36) resulted in the [CII] line being missed. In the case of J025-33 the [CII] line was located at the edge of our detection window missing some of the [CII] flux. Although Decarli et al. (2018) have presented ALMA [CII] observations of J025-33 (albeit with a lower angular resolution of $0 .{ }^{\prime \prime} 8$ ), in light of these issues we postpone discussion of the line measurements to future work.

\section{SMG AND QUASAR FIR SOURCE SIZES AND MORPHOLOGIES}

\subsection{WHDF source sizes and morphologies via CASA IMFIT}

In Figs. $2 \mathrm{a}$ and $2 \mathrm{~b}$ we show the FIR continuum emission detected in our ALMA observations of the WHDF SMGs and ATLAS $z>6$ quasars respectively. We measure the apparent and deconvolved sizes of the WHDF SMGs and ATLAS quasars by fitting Gaussians using the IMFIT $^{3}$ routine, which is part of the CASA (v4.7.2) package. As well as a measurement of the extent of the sources, IMFIT provides the integrated and peak flux (per beam) for each object which are used to calculate their SFR as detailed in Section 4. The measured sizes of the WHDF SMGs are given in Table 2. We see that all are resolved at our $0 .{ }^{\prime \prime} 1$ resolution.

In Fig. 3 we compare the ALMA profiles of the WHDF sources with the profiles of their counterpart detections from a $0 .{ }^{\prime \prime} 1$ resolution HST Advanced Camera for Surveys (ACS) $i$-band imaging. Fig. 4 shows a comparison of the ALMA profiles of the WHDF sources with their counterpart profiles from the H-band imaging of Metcalfe et al. (2006) with a seeing of $0 .{ }^{\prime \prime} 9$, obtained with the Calar Alto Omega Prime camera (Bizenberger et al. 1998). The measured ALMA, $i$ and $H$-band profiles presented in these figures are all obtained using the IRAF (v2.16.1) IMEXAMINE ${ }^{4}$ routine and we fit these profiles with Gaussian functions using a non-linear least squares fitting technique with the SCIPY (v0.17.0; Virtanen et al. 2020) 'curve_fit' 5 module. In Table 2 we also list the HST $i$-band and PSF-corrected, Calar Alto $H$-band FWHM of Gaussian profiles of the WHDF SMGs.

Next, we describe the results for each target in turn, particularly featuring their sub-mm + optical extents and morphologies, complementing the treatment of Paper I where the ALMA observations were mainly used to identify multi-wavelength counterparts to facilitate SED fitting:

LAB-01 6: This source has a HST-i+SPIES MIR counterpart classed in Paper I as a quasar at $z=2.6$ based on detection of weak X-rays and the optical-MIR SED fit. The SED fit also gave $A_{V}=1.75 \pm 0.25 \mathrm{mag}$ and dust temperature, $T=40 \pm 6 K$. Figs. 3,4 indicate that the

\footnotetext{
3 https://casa.nrao.edu/docs/taskref/imfit-task.html

4 https://imexam.readthedocs.io/en/latest/imexam/iraf_ imexam.html

5 https://docs.scipy.org/doc/scipy/reference/generated/

scipy.optimize.curve_fit.html

6 the SIMBAD IDs for all these objects follow the "[BHM2012] WHDFLAB-01" SIMBAD ID naming convention.
}

counterpart is classed as a galaxy in HST-i with FWHM $1.8 \mathrm{kpc}$ and unresolved in ground-based $\mathrm{H}$ band. The ALMA image in Fig. 2(a) is reasonably symmetric with deconvolved image FWHM axis sizes of $1.4 \times 1.1 \mathrm{kpc}$. LAB- 01 thus has an $\approx 1.47 \times$ larger extent in the $i$-band compared to its ALMA FIR continuum extent. The $870 \mu \mathrm{m}$ contours show no other low $\mathrm{S} / \mathrm{N}$ features.

LAB-02: The optical counterpart to this source was classed as being consistent with a quasar at $z=3.1 \pm 0.25$ on the basis of the SED fit with $A_{V}=0.25 \pm 0.38 \mathrm{mag}$ and $T=39 \pm 9 K$. Fig. 3 shows that the counterpart is resolved with FWHM $=1.5 \mathrm{kpc}$. This compares to the elongated $2.3 \times 0.8 \mathrm{kpc}$ of the sub-mm source which lies at $\approx 7$ $\mathrm{kpc}$ NE of the HST $i$ source. There is also an $8.4 \mathrm{GHz}$ radio source at $\approx 22 \mathrm{kpc}$ from the sub-mm source. Clearly, in the sub-mm we are seeing a dusty disc that may be small enough to be classed as a bar, possibly similar to those seen by Hodge et al. (2019) in some ALESS sources, although other interpretations cannot be ruled out e.g. a merger or a lensed galaxy. But again no spiral features are visible. This may be consistent with dust heating from the nucleus affecting features at a few kpc radius. No X-ray emission was detected at the sub-mm source position to a limit of $\approx 2 \times 10^{-17} \mathrm{ergs} \mathrm{cm}^{-2} \mathrm{~s}^{-1}$ in the $0.5-2 \mathrm{keV}$ band which could still be consistent with the presence of an AGN absorbed by an edge-on disc. Indeed, no optical, NIR nor MIR flux was detected at the sub-mm position and all may be similarly absorbed. This might suggest that the HST $i$ source at $\approx 7 \mathrm{kpc}$ is a companion rather than the counterpart, as assumed here, and this has to be borne in mind in noting that the HST $i$ and ALMA images show similar sizes in Fig. 3. The actual sub-mm source may be obscured by much more than the $A_{V}=0.25$ mag estimated for the source; a Compton thick source could be obscured by $A_{V}>1000$ mag and explain the lack of X-ray and even $4.6 \mu \mathrm{m}$ detection.

LAB-03: With just a marginal detection in the $H$-band at the ALMA position, no counterpart was claimed for this SMG, only a companion at $1 .{ }^{\prime \prime} 82$ distance from the sub-mm source. The SED fit for the companion implied $z=2.7 \pm 0.35$ and assuming both are at the same redshift, they are separated on the sky by $\approx 15 \mathrm{kpc}$. Xray emission was detected at the SMG position, corresponding to $L_{\mathrm{X}}(1.2-2 \mathrm{keV}) \approx 1.1 \times 10^{43} \mathrm{erg} \mathrm{s}^{-1}$, and it was this that was crucial in its identification as a probable quasar ${ }^{7}$. The sub-mm source has elongation between that of LAB-01 and LAB-02 with extent $1.8 \mathrm{kpc} \times 1.1 \mathrm{kpc}$. Again the difficulty in finding an optical or MIR counterpart could be due to significant dust absorption and this might be related to being seen at an angle rather than face-on. No low surface brightness sub-mm features were detected.

LAB-04: Although SED fitting marginally preferred a star-forming galaxy template, the counterpart was detected in X-rays, with a luminosity corresponding to $L_{\mathrm{X}}(1.2-2 \mathrm{keV}) \approx 1.8 \times 10^{43} \mathrm{erg} \mathrm{s}^{-1}$ at $z \approx 3$, and its red [3.6]-[4.5] colour was also consistent with it being a quasar. The AGN SED fit gave $z=3.0$ and $A_{V}=2.5 \mathrm{mag}$, in line with its lack of UBRI detection. At this redshift, its deconvolved extent is $1.4 \mathrm{kpc} \times 1.0 \mathrm{kpc}$ so again its sub-mm morphology is compact and reasonably symmetric. LAB-04 has a detection in the $H$-band with an $\approx 16 \times$ larger FWHM extent $(\approx 18 \mathrm{kpc})$ relative to the ALMA profile of this source ( $\approx 1.2 \mathrm{kpc}$, see Table 3 and Fig. 4$)$.

LAB-05: This object is identified as an X-ray absorbed quasar at a spectroscopic redshift of $z=2.12$, and SED fitting gave $A_{V}=1.0$

${ }^{7}$ Fig. 16 of Luo et al. (2017) shows that in their analysis of the Chandra 7 MSec observations of Chandra Deep Field-South that $L_{X}(0.5-7 \mathrm{keV})<3 \times$ $10^{42} \mathrm{ergs} \mathrm{s}^{-1}$ is already a conservative upper limit on the X-ray luminosity of a star-forming galaxy due to Low-Mass X-ray Binaries, with very few examples of star-forming galaxies being seen above the usual quasar limit of $L_{\mathrm{X}}>1 \times 10^{42} \mathrm{ergs} \mathrm{s}^{-1}$ (e.g. Zezas et al. 1998; Moran et al. 1999). 

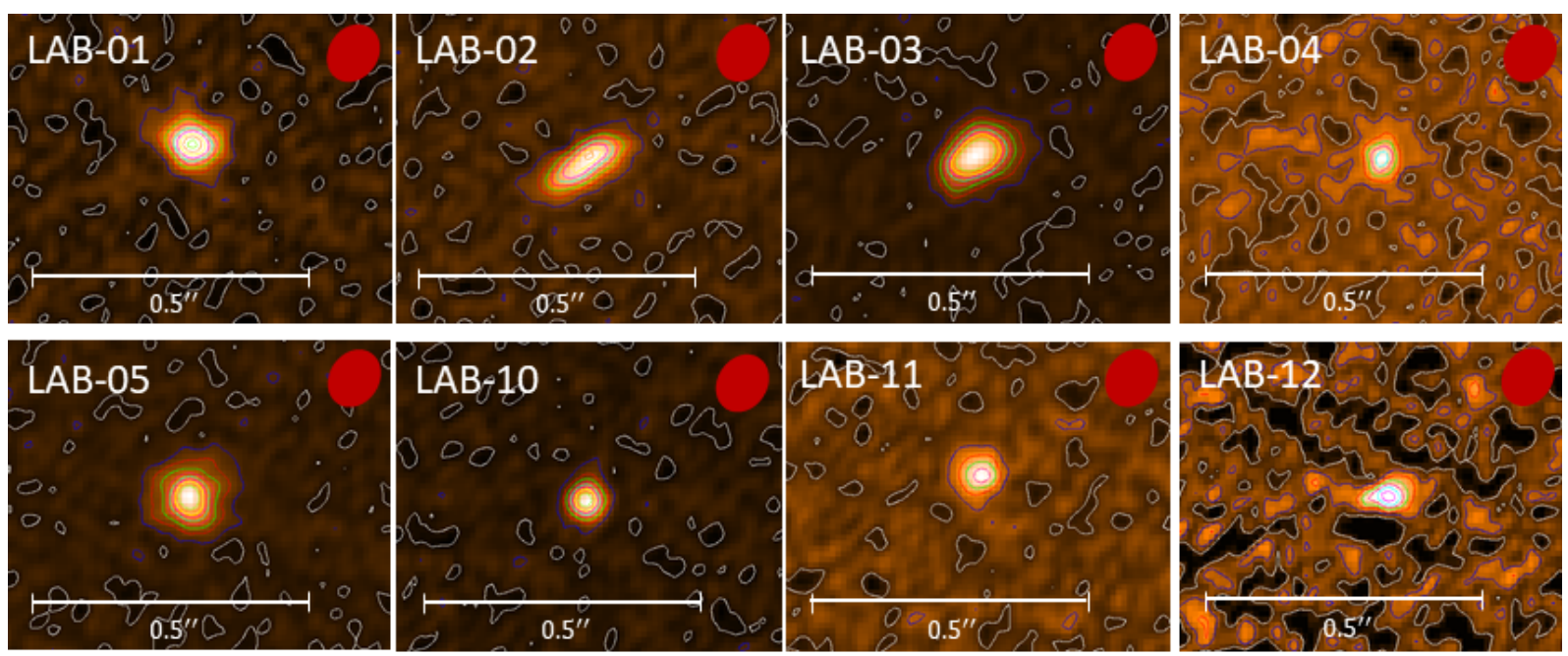

(a)
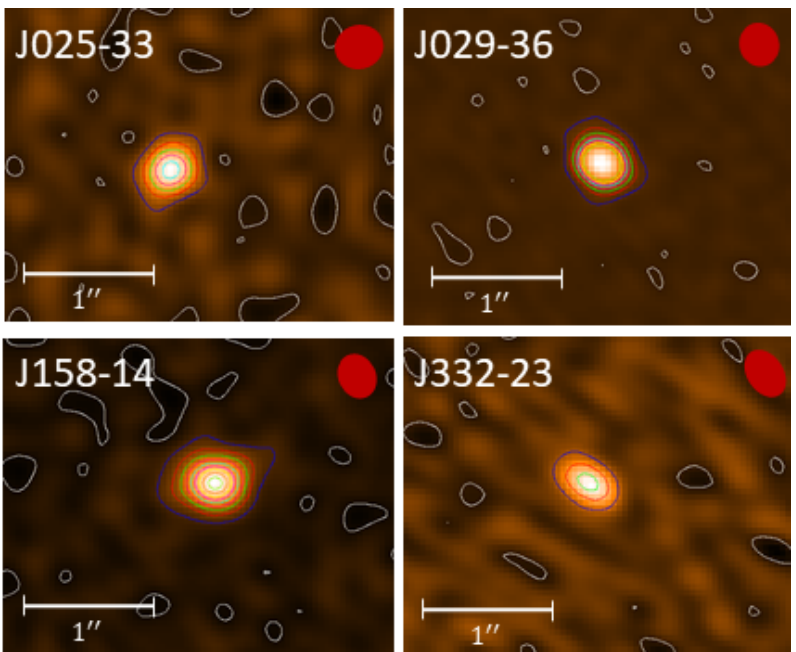

(b)
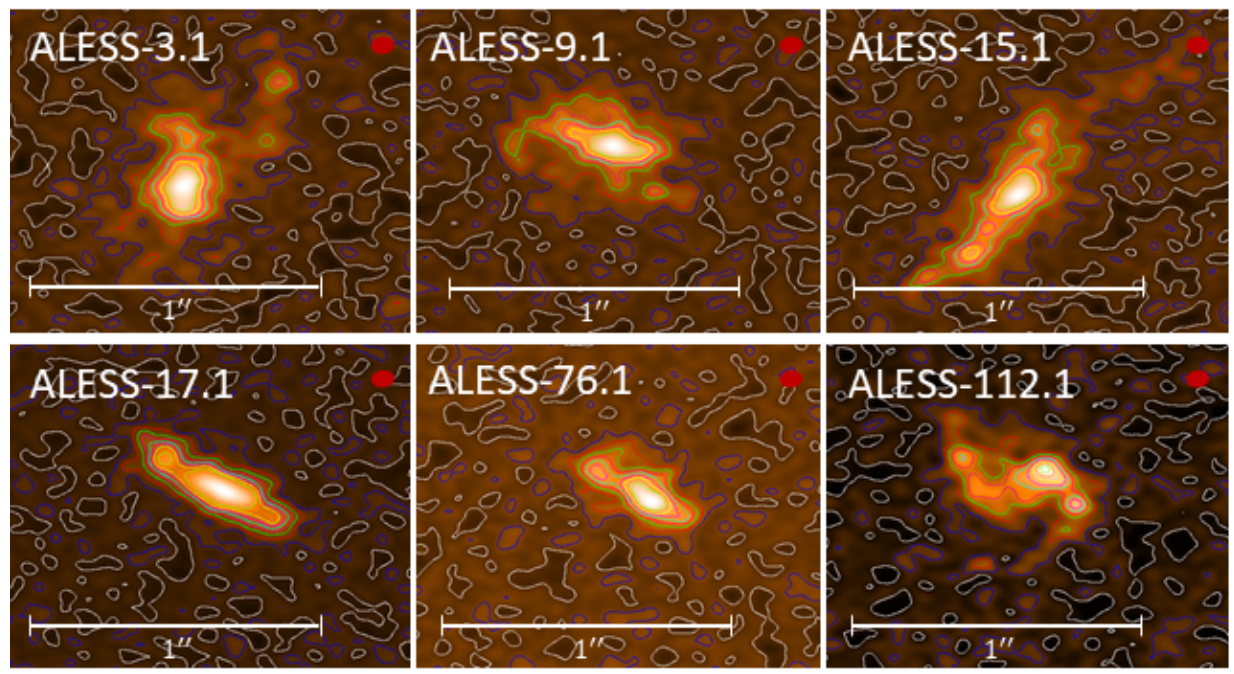

(c)

Figure 2. (a) ALMA 'thumbnails' with a resolution of 0. " 1 , showing the continuum emitting regions of the WHDF sub-mm sources. (b) ALMA 'thumbnails' with a resolution of $0 . " 3$, showing the continuum emitting regions of the $z \approx 6$ ATLAS quasars. (c) 0. " 07 resolution ALMA "thumbnails showing the continuum emission and hints of sub-structure detection in the ALESS SMGs of Hodge et al. (2019). In all cases, the contour levels are kept consistent to allow for direct visual comparison of the extent of the sources and the beams are shown by the red ellipses. 
Table 1. Summary of WHDF SMG and $z>6$ QSO properties.

\begin{tabular}{|c|c|c|c|c|c|c|c|}
\hline Name & $\begin{array}{c}\text { Short Name } \\
\text { (2) }\end{array}$ & $\begin{array}{c}\text { RA } \\
(\mathrm{J} 2000) \\
(3)\end{array}$ & $\begin{array}{l}\text { Dec } \\
\text { (4) }\end{array}$ & $\begin{array}{l}z \\
(5)\end{array}$ & $\begin{array}{c}M_{1450 \AA} \\
\text { (Mag) } \\
\text { (6) }\end{array}$ & $\begin{array}{c}M_{\mathrm{BH}}\left[\mathrm{C}_{\mathrm{IV}}\right] \\
\left(10^{9} \mathrm{M}_{\odot}\right) \\
(7)\end{array}$ & $\begin{array}{c}\text { Reference(s) } \\
\text { (8) }\end{array}$ \\
\hline WHDF-LAB-01 & LAB-01 & $00: 22: 37.58$ & $+00: 19: 18.4$ & $2.60 \pm 0.15$ & - & - & 3,4 \\
\hline WHDF-LAB-02 & LAB-02 & $00: 22: 28.44$ & $+00: 21: 47.6$ & $3.10 \pm 0.25$ & - & - & 3,4 \\
\hline WHDF-LAB-03 & LAB-03 & $00: 22: 45.96$ & $+00: 18: 41.2$ & $2.70 \pm 0.35 \dagger$ & - & - & 3,4 \\
\hline WHDF-LAB-04 & LAB-04 & $00: 22: 29.19$ & $+00: 20: 24.8$ & $3.00 \pm 0.60$ & - & - & 3,4 \\
\hline WHDF-LAB-05 & LAB-05 & $00: 22: 22.87$ & $+00: 20: 13.5$ & $2.12 \pm 0.03$ & - & - & 3,4 \\
\hline WHDF-LAB-10 & LAB-10 & $00: 22: 35.23$ & $+00: 24: 07.5$ & $0.90 \pm 0.20 \dagger$ & - & - & 3,4 \\
\hline WHDF-LAB-11 & LAB-11 & $00: 22: 24.84$ & $+00: 20: 11.4$ & $1.32 \pm 0.03$ & - & - & 3,4 \\
\hline WHDF-LAB-12 & LAB-12 & $00: 22: 25.48$ & $+00: 20: 06.6$ & $2.90 \pm 0.10$ & - & - & 3,4 \\
\hline VST-ATLAS J332.8017-32.1036 & J332-23 & $22: 11: 12.41$ & $-32: 06: 12.96$ & $6.32 \pm 0.03$ & $-26.79 \pm 0.06$ & 2.7 & 1 \\
\hline VST-ATLAS J158.6938-14.4211 & J158-14 & $10: 34: 46.51$ & $-14: 25: 15.96$ & $6.07 \pm 0.03$ & $-27.23 \pm 0.08$ & 2.4 & 1 \\
\hline VST-ATLAS J025.6821-33.4627 & J025-33 & $01: 42: 43.70$ & $-33: 27: 45.72$ & $6.31 \pm 0.03$ & $-27.50 \pm 0.06$ & 2.2 & 2 \\
\hline VST-ATLAS J029.9915-36.5658 & J029-36 & 01:59:57.96 & $-36: 33: 56.88$ & $6.02 \pm 0.03$ & $-26.97 \pm 0.08$ & 1.4 & 2 \\
\hline
\end{tabular}

Notes. (1) Source name (these names match the publications were the source names were first introduced; see references in column 8), (2) Short Name, (3) Right Ascension, (4) Declination, (5) Inferred Ly $\alpha$ redshift in case of ATLAS $z \approx 6$ quasars, and redshift based on AGN template SED fits presented in Table 2 of Paper I. $\dagger$ : redshifts estimated based on SED fits using optical/MIR detections of close companions to LAB-03 and LAB-10 since the direct counterpart of these sources are undetected in these bands. (6) $1450 \AA$ rest-frame absolute magnitude, (7) Black hole mass estimated from the Civ broad emission-line width, (8) References: 1.-Chehade et al. (2018); 2.-Carnall et al. (2015), 3.-Bielby et al. (2012), 4.-Paper I .

Table 2. IMFIT measurements of the WHDF SMG ALMA sub-mm sizes and fluxes.

\begin{tabular}{|c|c|c|c|c|c|c|c|c|}
\hline Object & $\begin{array}{c}\text { Major axis } \\
\text { FWHM } \\
{\left[{ }^{\prime \prime}\right]} \\
(2)\end{array}$ & $\begin{array}{c}\text { Minor axis } \\
\text { FWHM } \\
{\left[{ }^{\prime \prime}\right]} \\
(3)\end{array}$ & $\begin{array}{c}\text { Major axis } \\
\text { FWHM } \dagger \\
{\left[{ }^{\prime \prime}\right]} \\
(4)\end{array}$ & $\begin{array}{c}\text { Minor axis } \\
\text { FWHM } \dagger \\
{\left[^{\prime \prime}\right]} \\
(5)\end{array}$ & $\begin{array}{c}\text { Major axis } \\
\mathrm{FWHM}_{\dagger} \\
(\mathrm{kpc}) \\
(6)\end{array}$ & $\begin{array}{c}\text { Minor axis } \\
\text { FWHM } \dagger \\
(\mathrm{kpc}) \\
(7)\end{array}$ & $\begin{array}{l}\text { Integrated } \\
\text { flux density } \\
(\mathrm{mJy}) \\
(8)\end{array}$ & $\begin{array}{c}\text { Peak flux } \\
\text { density } \\
\text { (mJy/beam) } \\
\text { (9) }\end{array}$ \\
\hline LAB-01 & $0.20 \pm 0.008$ & $0.17 \pm 0.006$ & $0.18 \pm 0.010$ & $0.14 \pm 0.009$ & $1.4 \pm 0.08$ & $1.1 \pm 0.07$ & $3.84 \pm 0.18$ & $1.10 \pm 0.04$ \\
\hline LAB-02 & $0.32 \pm 0.013$ & $0.14 \pm 0.004$ & $0.31 \pm 0.014$ & $0.10 \pm 0.006$ & $2.4 \pm 0.11$ & $0.8 \pm 0.05$ & $4.68 \pm 0.19$ & $1.04 \pm 0.04$ \\
\hline LAB-03 & $0.25 \pm 0.006$ & $0.16 \pm 0.004$ & $0.22 \pm 0.007$ & $0.13 \pm 0.004$ & $1.8 \pm 0.06$ & $1.1 \pm 0.03$ & $7.16 \pm 0.21$ & $1.76 \pm 0.04$ \\
\hline LAB-04 & $0.20 \pm 0.024$ & $0.16 \pm 0.017$ & $0.18 \pm 0.030$ & $0.12 \pm 0.026$ & $1.4 \pm 0.23$ & $1.0 \pm 0.20$ & $1.97 \pm 0.3$ & $0.61 \pm 0.07$ \\
\hline LAB-05 & $0.21 \pm 0.008$ & $0.20 \pm 0.008$ & $0.18 \pm 0.012$ & $0.17 \pm 0.012$ & $1.5 \pm 0.10$ & $1.4 \pm 0.10$ & $5.46 \pm 0.26$ & $1.31 \pm 0.05$ \\
\hline LAB-10 & $0.14 \pm 0.004$ & $0.12 \pm 0.004$ & $0.094 \pm 0.008$ & $0.074 \pm 0.009$ & $0.7 \pm 0.06$ & $0.6 \pm 0.07$ & $2.30 \pm 0.10$ & $1.33 \pm 0.04$ \\
\hline LAB-11 & $0.17 \pm 0.011$ & $0.16 \pm 0.010$ & $0.14 \pm 0.018$ & $0.13 \pm 0.019$ & $1.1 \pm 0.15$ & $1.1 \pm 0.16$ & $1.51 \pm 0.12$ & $0.55 \pm 0.03$ \\
\hline LAB-12 & $0.20 \pm 0.024$ & $0.10 \pm 0.008$ & $0.17 \pm 0.030$ & $0.036 \pm 0.021$ & $1.3 \pm 0.23$ & $0.3 \pm 0.16$ & $1.68 \pm 0.24$ & $0.83 \pm 0.08$ \\
\hline
\end{tabular}

Notes. (1) Source short name, (2) Major axis FWHM of the continuum emitting region, (3) Minor axis FWHM, (4) Major axis FWHM of the continuum emitting region deconvolved from beam, (5) Minor axis deconvolved FWHM, (6) Major axis deconvolved FWHM (kpc), (7) Minor axis deconvolved FWHM (kpc), (8) IMFIT integrated flux densities, (9) IMFIT peak flux densities. $\dagger$ represents extents deconvolved from beam/PSF.

mag. Table 2 shows ALMA extents of $1.4 \mathrm{kpc} \times 1.2 \mathrm{kpc}$ and so this source is barely resolved. The HST $i$ image in Fig. 1 of Paper I shows that LAB-05 has a blotchy appearance with several possible nuclei. Despite the dust absorption, the quasar shows a strong ultraviolet excess with $(\mathrm{U}-\mathrm{B})_{\text {Vega }}=-1.25 \mathrm{mag}$ (Heywood et al. 2013). The absorbed X-ray source and the sub-mm source are reasonably coincident with one of these nuclei that is slightly offset with respect to the $i$-band image. We see that the size of the $i$-band nucleus is similar in size to the sub-mm image at $\approx 1.0-1.4 \mathrm{kpc}$. But the full extent of the host galaxy is larger $(\approx 2.7 \times)$ and resolved even on the $0 .{ }^{\prime \prime} 9$ scale of the $H$-band image with a deconvolved extent of $0 . " 5$ or $\approx 4 \mathrm{kpc}$. Thus the sub-mm source seems highly compact and associated directly with the AGN. This looks similar to what is seen in SMGs LAB-01, LAB-02 and LAB-04.

LAB-10: This sub-mm source also showed no direct counterpart in any band other than 250 and $350 \mu \mathrm{m}$ and its redshift of $z=0.9$ was estimated from a companion at $\approx 9 \mathrm{kpc}$ separation. Assuming this redshift, LAB-10 is $\approx 0.7 \mathrm{kpc}$ in extent (see Table 3 ) and again highly compact and barely resolved with no evidence of low surface brightness sub-mm features (see Fig. 2c). Otherwise, with no counterpart no further conclusion can be drawn except that the host must be highly obscured.

LAB-11: This sub-mm source is identified with a $z=1.32 \mathrm{X}$-ray absorbed quasar and SED fitting gives $A_{V}=1.5 \pm 0.25 \mathrm{mag}$ and dust temperature $T=41 \pm 10 \mathrm{~K}$. The X-ray source is coincident with the sub-mm source. However, the quasar X-ray absorption is much higher than implied by the fitted dust absorption. Despite the dust absorption, the quasar still shows some ultra-violet excess with (UB) Vega $=-0.72 \mathrm{mag}$ (Heywood et al. 2013). There is also an 8.4 GHz radio source at 1." 2 from the ALMA position.

The optical structure of the LAB-11 host galaxy is clearly resolved at HST $i$ - band resolution with an extent of $\approx 3 \mathrm{kpc}, \approx 3 \times$ larger than the $1.1 \mathrm{kpc} \times 1.1 \mathrm{kpc}$ of the sub-mm source which is barely resolved at $0 .{ }^{\prime \prime} 1$ ALMA resolution. Fig. 1 of Paper I shows that the 


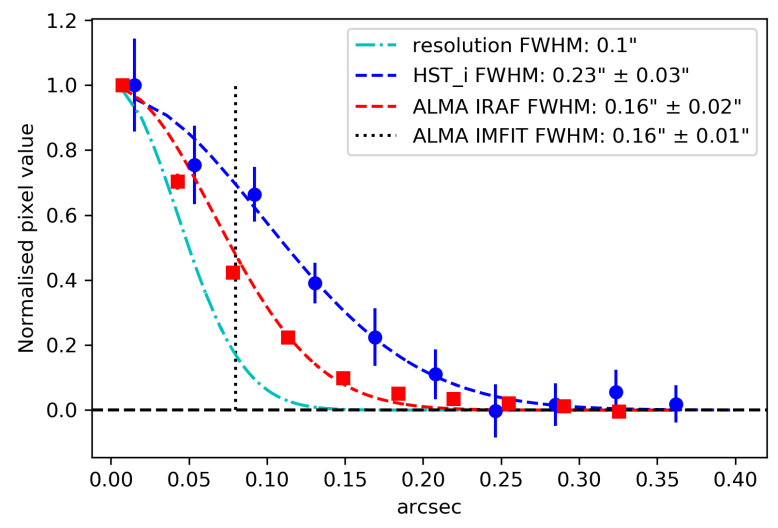

(a) LAB-01

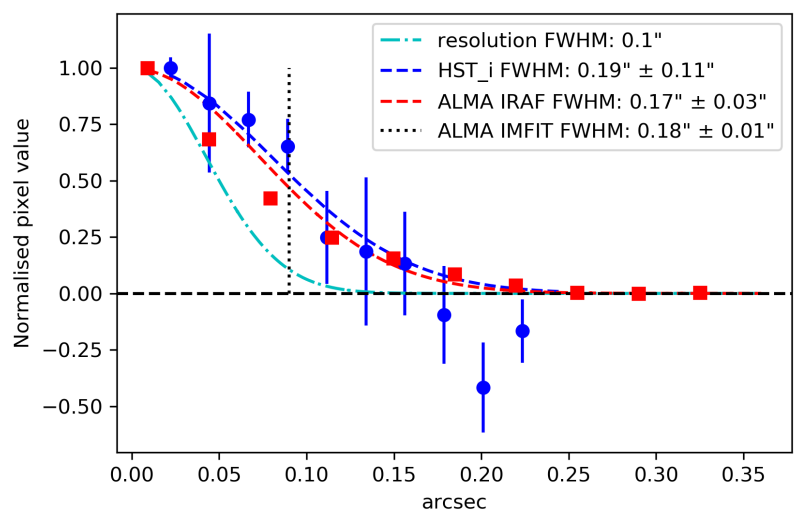

(c) LAB-05

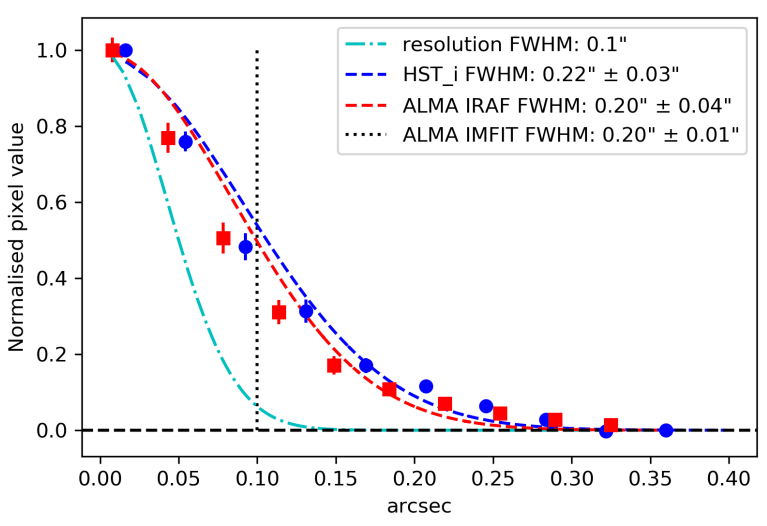

(b) LAB-02

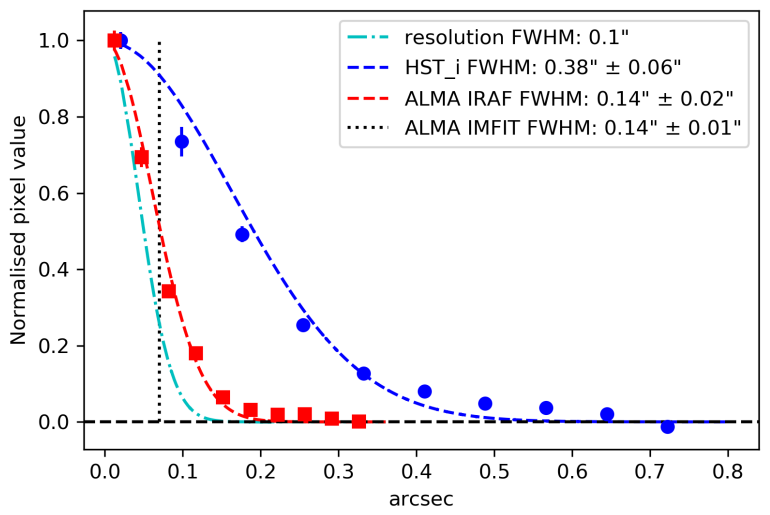

(d) LAB-11

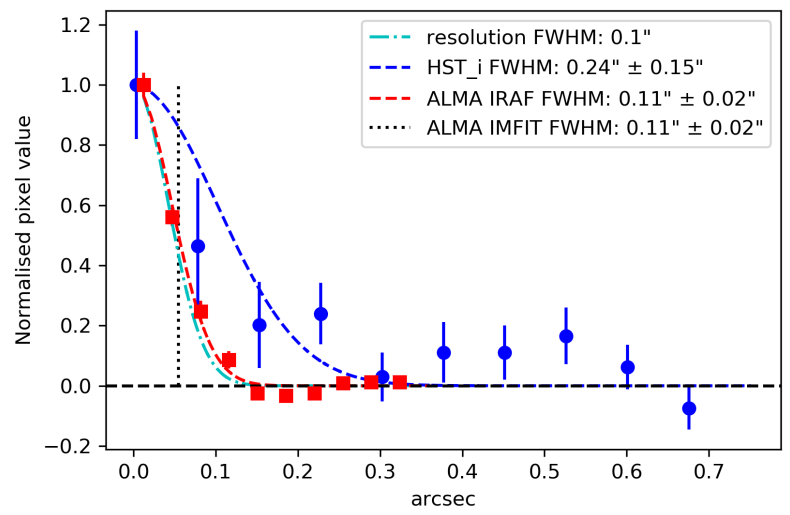

(e) LAB-12

Figure 3. Comparison of ALMA and HST $i$-band profiles of LABOCA sources with detections in both bands. For comparison we include a Gaussian with a $0 . " 1$ FWHM indicating the resolution of the HST and ALMA imaging. Here, no PSF correction has been applied to the ALMA or HST profiles. The range of redshifts covered here is $1.32<z<3.1$ so the observed $i$-band corresponds to the rest wavelength range 2100-3700 which seems acceptably narrow. Indeed, excluding LAB-11 this range reduces still further to $2700-3700 \AA$, arguing that these $i$-band profiles can be consistently compared.

host galaxy of LAB-11 has a smoother, less nucleated structure than the LAB-05 quasar host. Fig. 3 shows that LAB-11 is clearly detected in the $i$-band where it has an extent $\approx 3 \times$ larger than its counterpart ALMA detection, while it is only marginally resolved in the $H$-band (see Fig. 4 and Table 3).
LAB-12: This sub-mm source was detected for the first time in the ALMA observation at $10 .{ }^{\prime \prime} 6$ from LAB-11 and is not in the flux limited sample. Nevertheless, an optical+MIR counterpart was detected and SED fitting and MIR colour were consistent with it being a probable quasar at $z=2.9$. Its sub-mm structure appears 


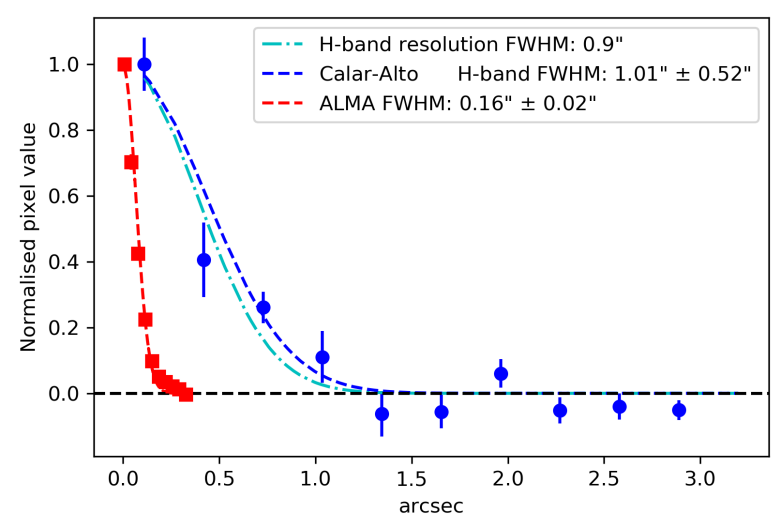

(a) LAB-01

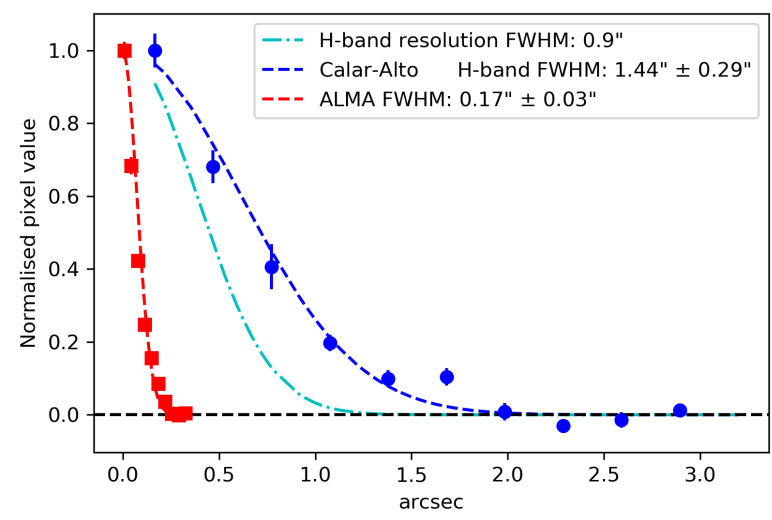

(c) LAB-05

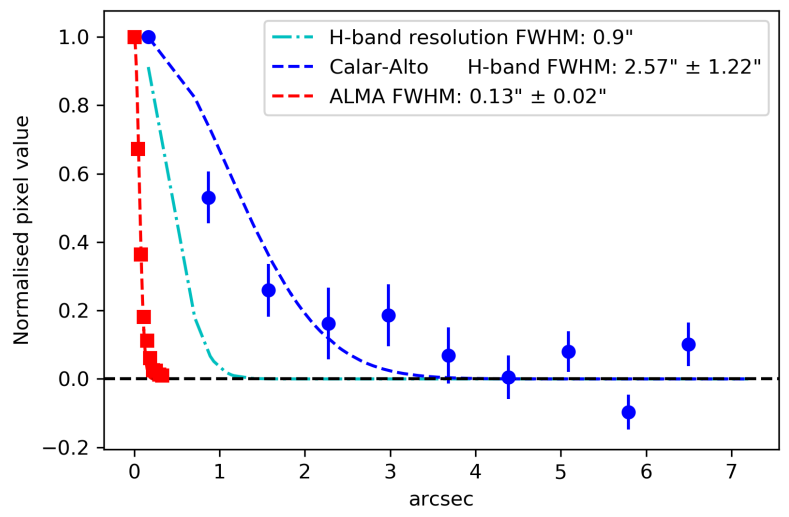

(b) LAB-04

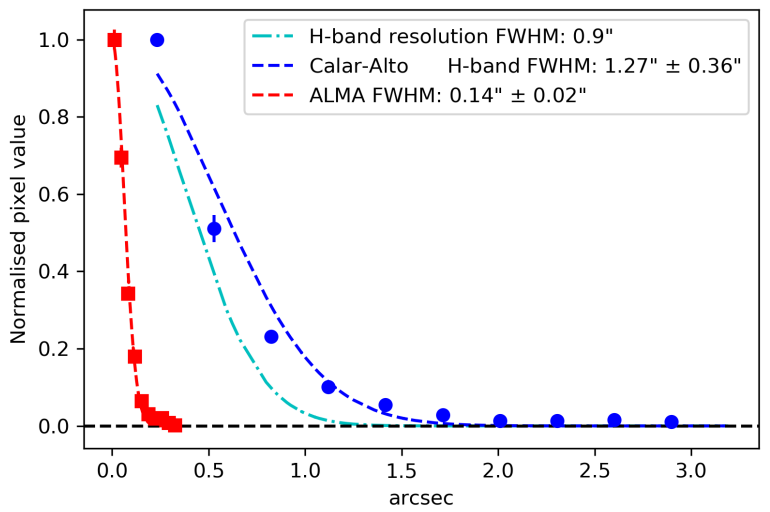

(d) LAB-11

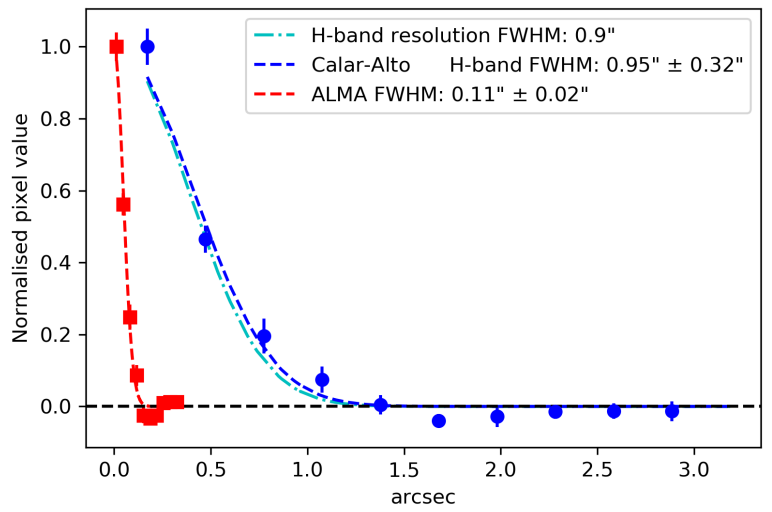

(e) LAB-12

Figure 4. Comparison of ALMA and Calar Alto $H$-band profiles of the WHDF sources with detections in both bands. For comparison, we include a Gaussian with a $0 . " 9$ FWHM indicating the resolution of the $H$-band imaging. Note that unlike in Cols. 6 and 7 of Table 3 the $H$-band source sizes in the legends are not corrected for the $0 . " 9$ seeing PSF. Similarly, no PSF correction has been applied to the ALMA profiles. The range of redshifts covered here is $1.32<z<3.0$ so the observed $H$-band corresponds to the rest wavelength range 4000-6900 $\AA$ which seems acceptably narrow. Indeed, excluding LAB-11 this range reduces still further to $4000-5100 \AA$, arguing that these $H$-band profiles can be consistently compared.

elongated with extents of $1.3 \mathrm{kpc} \times 0.3 \mathrm{kpc}$ and Fig. 1 of Paper I shows that the HST $i$-band counterpart has a similar elongation but with $\approx 2 \times$ larger scale (see also Fig. 3 and Table 3 ). So in this lower luminosity sub-mm source, the sub-mm and optical morphologies and scale look more similar to what naively might be expected if the dust was heated more by in situ star-formation. 
Table 3. IMFIT+IRAF measurements of the WHDF SMG sizes in sub-mm, $i$ and $H$ bands and their ratios.

\begin{tabular}{ccccccc|cc}
\hline Object & $\begin{array}{c}\text { FIR Major } \\
\text { FWHM } \dagger\end{array}$ & $\begin{array}{c}\text { FIR Minor } \\
\text { FWHM } \dagger\end{array}$ & $\begin{array}{c}i \text {-band } \\
\text { FWHM }\end{array}$ & $\begin{array}{c}H \text {-band } \\
\text { FWHM }\end{array}$ & $\begin{array}{c}i \text {-band } \\
\text { FWHM } \dagger\end{array}$ & $\begin{array}{c}H \text {-band } \\
\text { FWHM } \dagger\end{array}$ & $\begin{array}{c}\text { i-band/FIR } \\
\text { ratio }\end{array}$ & $\begin{array}{c}H \text {-band/FIR } \\
\text { ratio }\end{array}$ \\
\hline LAB-01 & $0.18 \pm 0.010$ & $0.14 \pm 0.009$ & $0.23 \pm 0.03$ & $1.01 \pm 0.52$ & $0.21 \pm 0.020$ & $0.46 \pm 0.52$ & $1.34 \pm 0.13$ & $2.92 \pm 1.14$ \\
LAB-02 & $0.31 \pm 0.014$ & $0.10 \pm 0.006$ & $0.22 \pm 0.03$ & - & $0.20 \pm 0.020$ & - & $0.98 \pm 0.11$ \\
LAB-03 & $0.22 \pm 0.007$ & $0.13 \pm 0.004$ & - & - & - & - & - \\
LAB-04 & $0.18 \pm 0.030$ & $0.12 \pm 0.026$ & - & $2.57 \pm 1.22$ & - & $2.40 \pm 1.22$ & - & - \\
LAB-05 & $0.18 \pm 0.012$ & $0.17 \pm 0.012$ & $0.19 \pm 0.011$ & $0.14 \pm 0.029$ & $0.16 \pm 0.11$ & $1.12 \pm 0.29$ & $0.92 \pm 0.63$ & $6.35 \pm 1.69^{*}$ \\
LAB-10 & $0.094 \pm 0.008$ & $0.074 \pm 0.009$ & - & - & - & - & - \\
LAB-11 & $0.14 \pm 0.018$ & $0.13 \pm 0.019$ & $0.38 \pm 0.060$ & $1.27 \pm 0.36$ & $0.37 \pm 0.060$ & $0.90 \pm 0.36$ & $2.80 \pm 0.60$ & $-6.79 \pm 2.89$ \\
LAB-12 & $0.17 \pm 0.030$ & $0.036 \pm 0.021$ & $0.24 \pm 0.15$ & $0.95 \pm 0.32$ & $0.22 \pm 0.15$ & $0.30 \pm 0.32$ & $2.16 \pm 1.59$ & $3.01 \pm 3.26$ \\
\hline
\end{tabular}

Notes. All columns except the last two are in units of arcseconds. $\dagger$ represents extents deconvolved from beam/PSF. *LAB-5 is associated with a multiply nucleated host galaxy which is resolved into separate objects in the $i$-band but not the $H$-band. In this case, the $i$-band size is measured from the nucleus nearest the SMG and the $H$-band size is measured for the entire host galaxy.

\subsection{Comparison with ALESS SMG sizes and morphologies}

For comparison, in Fig. $2 c$ we plot high resolution $\left(\approx 0 .{ }^{\prime \prime} 07\right)$ ALMA observations of the six luminous SMGs from the ALESS sample (Hodge et al. 2013, 2016) studied by Hodge et al. (2019). We chose to compare to these data because their combination of ALMA high resolution and exposure time is more comparable to our WHDF data than for other samples such as those of e.g. Ikarashi et al. (2017), Elbaz et al. (2018) or Gullberg et al. (2019). The ALESS flux density range also overlaps our WHDF range more than the fainter submm galaxy samples from Rujopakarn et al. (2016, 2019), Franco et al. (2020) or ?. Fig. 2c shows that unlike the WHDF SMGs, the ALMA observations of ALESS SMGs reveal potential evidence of sub-structure detection in these sources. Furthermore, the ALESS SMGs appear to have half-light radii $^{8}$ that are on average $\approx 3 \times$ larger than those of the WHDF SMGs. The average ALESS SMG flux density is also $\approx 2 \times$ brighter than that of the WHDF SMGs. Given the factor of $\approx 2$ lower exposure time and lower resolution $\left(0 .{ }^{\prime \prime} 1 \mathrm{vs}\right.$ $\left.0 .{ }^{\prime \prime} 07\right)$ of the ALMA observations of the WHDF sources compared to those of the ALESS SMGs, it might be thought that sub-structure currently remains undetected in the WHDF observations. However, the average surface brightness of $\approx 200 \mu \mathrm{Jy} /$ beam is clearly reached by all the WHDF sub-mm observations (see Table 4). The two highest noise cases are LAB-04 and LAB-12 with $\pm 70-80 \mu \mathrm{Jy} /$ beam and even they would detect the ALESS diffuse structure at $2.5-2.8 \sigma$ over just one beam size and clearly more if averaged over a large area. These significances of detection would rise to $\approx 5-6 \sigma$ for the other WHDF SMGs.

Another possibility, however, is that despite the fact that both samples occupy a similar redshift range, there is an inherent difference between the two SMG populations. While the WHDF SMGs were initially detected in blind LABOCA observation of the WHDF, the ALESS SMGs studied by Hodge et al. (2019) were selected as the brightest amongst the large ALESS sample (see Hodge et al. 2013; Hodge et al. 2016). This selection could in turn bias the sample towards selecting larger than average SMGs that are more likely to contain distinct sub-structures. This would also be consistent with previous claims of a slow increase in SMG FIR size with FIR luminosity (e.g. Fujimoto et al. 2017; Gullberg et al. 2019).

\footnotetext{
8 The half-light radius of a source is defined as the mean of its major and
} minor axes' FWHM/2.

\subsection{Comparison with $z>6$ quasar sizes and morphologies}

We also analysed our four $z>6$ quasars using IMFIT in the same way as for the WHDF SMGs. The resulting sub-mm images are shown in Fig. $2 b$ and their sizes in Table 5. With the exception of J025-33, all our other sources appear to be resolved. We now discuss each quasar individually.

J332-23 The sub-mm counterpart of this $z=6.32$ quasar is just resolved by ALMA at $0 .{ }^{\prime \prime} 4 \times 0 .{ }^{\prime \prime} 28$ resolution. It has an apparently elongated sub-mm morphology with deconvolved extents measured as $0.9 \times 0.5 \mathrm{kpc}$. However, the beam is also elongated for this observation and this means large errors on these deconvolved sizes. The longer deconvolved axis is detected at $2.5 \sigma$ and the shorter axis is detected at $1.7 \sigma$. However, the evidence against circularity is only significant at $0.8 \sigma$. We conclude that this image is resolved but with only marginal evidence for elongation. We note that a second unidentified sub-mm source, with integrated and peak flux densities $0.66 \pm 0.055 \mathrm{mJy}$ and $0.45 \pm 0.024 \mathrm{mJy}$, is detected by ALMA at $5 . " 4 \mathrm{SW}$ from the source associated with the quasar.

J158-14 The sub-mm counterpart of this $z=6.07$ quasar is again indicated as resolved by IMFIT with deconvolved extents of $1.1 \mathrm{kpc}$ $\times 0.8 \mathrm{kpc}$. Again there is no indication against a symmetric, circular sub-mm image, given the errors on these extents quoted in Table 5.

J025-33 at $z=6.31$ was identified as a point source in the sub-mm by IMFIT with a FWHM major axis $<0 .{ }^{\prime \prime} 2 \times 0 .{ }^{\prime \prime} 07$ giving an upper limit in physical size of $1.1 \mathrm{kpc} \times 0.4 \mathrm{kpc}$. In Fig. $2 \mathrm{~b}(\mathrm{~b}), \mathrm{J} 025-33$ shows a relatively circular shape, similar to the beam with its FWHM of $0 .{ }^{\prime \prime} 38 \times 0 .{ }^{\prime \prime} 34$ and IMFIT only gives an upper-limit $\left(<0 .{ }^{\prime \prime} 2\right.$ or $<1.0$ $\mathrm{kpc}$ ) on the extent of this source. Consequently, in Section 3 we only present a lower-limit for the star formation rate surface density $\left(\sum_{\mathrm{SFR}}\right)$ of this object. We note that J025-33 lies closer to the edge of the field in the ALMA observations and therefore has a lower $\mathrm{S} / \mathrm{N}$ compared to the other detections, which lie closer to the field centre.

J029-36 This is the most clearly resolved quasar with deconvolved sky extents of $0 .{ }^{\prime \prime} 33 \pm 0 .{ }^{\prime \prime} 02 \times 0 .{ }^{\prime \prime} 22 \pm 0 .{ }^{\prime \prime} 02$ which at $z=6.02$ translate to $1.9 \mathrm{kpc} \times 1.2 \mathrm{kpc}$. Again there is little evidence of noncircularity in the sub-mm image in Fig. 2 b or in Table 5.

Thus, helped by the angular diameter distance-redshift relation, $d_{\mathrm{A}}(z)$, we can resolve 3 out of $4 z>6$ quasar images despite the lower $\approx 0 . " 33$ ALMA resolution than for the WHDF SMGs. Assuming the upper limit for the unresolved extent of the J025-33 host we find an average of $1.25 \pm 0.22 \mathrm{kpc}$ for the average sub-mm size of the major axes of the quasar hosts in the sub-mm. This compares with $1.44 \pm 0.17 \mathrm{kpc}$ for the average of the major axes of the 8 WHDF 
Table 4. Noise levels (standard deviations) measured in $\mu \mathrm{Jy} / \mathrm{beam}$ in vicinity of ALMA targets.

\begin{tabular}{lccccccccccc}
\hline LAB-01 & LAB-02 & LAB-03 & LAB-04 & LAB-05 & LAB-10 & LAB-11 & LAB-12 & J332-23 & J158-14 & J025-33 & J029-36 \\
\pm 36.8 & \pm 38.6 & \pm 36.7 & \pm 68.8 & \pm 36.7 & \pm 35.3 & \pm 35.9 & \pm 79.7 & \pm 25.8 & \pm 24.7 & \pm 29.9 & \pm 31.4 \\
\hline
\end{tabular}

SMGs given in Table 2 which is statistically consistent with the $z>6$ quasar sizes after beam deconvolution.

The high redshift of the ATLAS $z>6$ QSOs makes any search for low surface brightness components more difficult than for the WHDF SMGs. Since there is typically a factor of $\approx 2$ difference in redshift the $(1+z)^{4}$ dimming law means that they will be $\approx 10 \times$ lower or $\approx 20 \mu \mathrm{Jy}$. An average of $\approx 100$ beam areas (i.e. only a $3^{\prime \prime} \times 3^{\prime \prime}$ extent) would be needed to detect them but none are seen. Although this result appears contrary to the FIR size-luminosity relation mentioned in Section 3.2, this might be due to the significantly higher redshift of these sources. At minimum, this means that our search for a diffuse component is at $\approx 150 \mu \mathrm{m}$ rest wavelength rather than $\approx 300 \mu \mathrm{m}$ for the ALESS/WHDF SMGs.

\section{SMG AND QUASAR FIR LUMINOSITIES AND SFR DENSITIES}

Following Decarli et al. (2018) and Beelen et al. (2006), we calculate the FIR Luminosity of our SMGs and quasars by modelling the dust continuum emission as a modified black body. Specifically, we estimate $L_{\text {FIR }}$ to the observed $S_{870 \mu \mathrm{m}}$ (WHDF SMGs) and $S_{1200 \mu \mathrm{m}}$ ( $z>6$ quasars) flux density using eqs (1) and (4) of Beelen et al. (2006). Assuming dust temperature $T_{\text {dust }}$ and with $v_{\mathrm{r}}$ the rest frequency corresponding to e.g. $870 \mu \mathrm{m}$ at redshift, $z$, we calculate $M_{\text {dust }}$ from:

$S_{870, \text { obs }}=\frac{1+z}{d_{\mathrm{L}}^{2}} M_{\text {dust }} B_{v_{\mathrm{r}}}\left(T_{\text {dust }}\right) \kappa\left(v_{\mathrm{r}}\right)$

where

$B_{v}\left(T_{\text {dust }}\right)=\frac{2 h v^{3}}{c^{2}} \frac{1}{e^{h v / k_{b} T_{\text {dust }}-1}}$,

is the Planck function and $\kappa(v)=0.077(v / 352 \mathrm{GHz})^{\beta} \mathrm{m}^{2} \mathrm{~kg}^{-2}$ is the opacity law, with dust emissivity index, $\beta=1.6$ (Beelen et al. 2006; Decarli et al. 2018). We then use this value for $M_{\text {dust }}$ to calculate $L_{\mathrm{IR}}$ from:

$L_{\mathrm{IR}}=4 \pi M_{\text {dust }} \int B_{v}\left(T_{\text {dust }}\right) \kappa(v) d v$

with the integral over frequencies, $v$, corresponding to rest wavelengths between 3-1100 $\mu$ m (following Kennicutt \& Evans 2012; Decarli et al. 2018). In this work, we set the dust temperature to the AGN dust temperature estimated in Paper I when available and otherwise $T_{\text {dust }}=35 \mathrm{~K}$ in the case of our WHDF sources (e.g. Cooke et al. 2018). We take $T_{\text {dust }}=47 \mathrm{~K}$ for our $z>6$ quasars (see e.g. Willott et al. 2017). We then obtain the SFR following Kennicutt \& Evans (2012):

$\frac{\mathrm{SFR}_{\mathrm{IR}}}{\mathrm{M}_{\odot} \mathrm{yr}^{-1}}=1.49 \times 10^{-10} \frac{L_{\mathrm{IR}}}{\mathrm{L}_{\odot}}$.

Following Hodge et al. (2019), we report two estimates of the star formation rate surface density, the integral or galaxy-averaged $\sum_{\mathrm{SFR}}$ and its peak value. The galaxy-averaged $\sum_{\mathrm{SFR}}$ is given by $\left(0.5 \times \mathrm{SFR}_{\mathrm{IR}}\right) /\left(\pi R_{e}^{2}\right)$ where $R_{e}$ is the half-light radius defined as in Section 3.2 as the mean of its major and minor axes' FWHM/2. The peak SFR density is calculated using the $S_{F R}$ IR value based on the peak flux density/beam and divided by the beam area defined from its major and minor axes at FWHM, $a, b$, as $\frac{\pi a b}{4 \ln (2)}$. Clearly, the closer a sub-mm source is to being unresolved the less difference there will be between the integrated and peak values and we shall see that this applies to many of the WHDF SMGs and $z>6$ quasars considered here.

\subsection{SFR densities and the SFR 'Eddington limit'}

The results of fitting the continuum emission maps of our sources using the IMFIT algorithm, are presented in Tables 2 and 5. The clean beam size for all WHDF observations was $0 .{ }^{\prime \prime} 11 \times 0 .{ }^{\prime \prime} 09$ while those for the $z>6$ quasars vary slightly as shown in Table 5. Also shown in both cases, are the beam deconvolved minor and major axes FWHM of the fits which are used to estimate the area of the continuum emitting regions. Also included are the integrated and peak flux densities of each source as measured by IMFIT.

Table 6 shows the dust mass, FIR luminosities, star formation rates and star formation rate surface densities of the WHDF and ATLAS $z \approx 6$ SMGs. We emphasise that the errors on these quantities may be underestimated because we have not included systematic errors such as the variation of the dust emissivity index, $\beta$, which may vary within sources as well as between sources. Similarly, dust temperatures are also assumed to be constant within individual sources and again this may turn out to be an over-simplification. Thus the absolute values of quantities such as the inferred dust mass for individual sources may be less reliable than inferred from the quoted statistical error. We should be on safer ground when we note e.g. that the dust masses for the 6 SMGs and the 6 quasars in Table 6 mostly lie within the narrow range $\approx 10^{8}-10^{9} \mathrm{M}_{\odot}$ although, even here, these assumptions have to be borne in mind. With this proviso, we further note that the FIR luminosities and star-formation rates also lie in the same ranges for the SMGs and quasars in Table 6 and we shall return to discuss these similarities further in Section 5.

In Fig. 5, we plot the integrated and peak $\sum_{S F R}$ values of our sources versus respectively the effective radius, $\mathrm{R}_{e}$, and average beam size, $(a+b) / 2$. For comparison, we also include measurements of the ALESS SMG sample, as presented in Fig. 6 of Hodge et al. (2019) where they are compared to a 'lower bound' to the 'Eddington limit' of SFR surface density, as derived in Section 4.1 of Hodge et al. (2019). This lower bound was obtained by converting the Eddington flux for optically thick starbursts (as given by Andrews \& Thompson 2011) to the Eddington limited SFR density: $\left(\sum_{\mathrm{SFR}}\right)_{\mathrm{Edd}} \approx 7.2 \mathrm{M}_{\odot} \mathrm{yr}^{-1} \mathrm{kpc}^{-2} f_{\mathrm{gas}}^{-1 / 2} f_{\mathrm{dg}}^{-1}$. Here, the gas fraction $f_{\text {gas }}$ is taken to be unity as the most extreme scenario, while the dust-to-gas ratio $f_{\mathrm{dg}}$ is assumed to be $1 / 90$, as proposed for SMGs by Magnelli et al. (2012); Swinbank et al. (2014). It is this first assumption that makes the value they derived, $\sum_{\mathrm{SFR}} \approx 650 \mathrm{M}_{\odot} \mathrm{yr}^{-1} \mathrm{kpc}^{-2}$, a lower bound for this quantity. Note that in Fig. 5, the SFR surface density values for the 6 ALESS SMGs have been re-calculated according to eqs 1-4 for consistency with our SMG and quasar values. We find these recalculated values for the integrated SFR densities are a factor of $2.1 \pm 0.23$ higher than those given by Hodge et al. (2019) due to 
Table 5. IMFIT measurements of the $z>6$ quasar ALMA FIR source sizes and fluxes.

\begin{tabular}{lccccccc}
\hline Object & $\lambda$ & Beam size & $\begin{array}{c}\text { Major axis } \\
\text { FWHM } \\
{\left[{ }^{\prime \prime}\right]}\end{array}$ & $\begin{array}{c}\text { Minor axis } \\
\text { FWHM } \\
{\left[{ }^{\prime \prime}\right]}\end{array}$ & $\begin{array}{c}\text { Area } \\
\left(\mathrm{kpc}^{2}\right)\end{array}$ & $\begin{array}{c}\text { Integrated flux } \\
\text { density } \\
(\mathrm{mJy})\end{array}$ & $\begin{array}{c}\text { Peak flux } \\
\text { density } \\
(\mathrm{mJy} / \mathrm{beam}) \\
(8)\end{array}$ \\
\hline $\mathrm{J} 332-23$ & 1202.27 & $0.40 \times 0.28$ & $0.16 \pm 0.07$ & $0.09 \pm 0.05$ & $0.3 \pm 0.2$ & $0.47 \pm 0.03$ & $0.41 \pm 0.02$ \\
$\mathrm{~J} 158-14$ & 1150.06 & $0.36 \times 0.29$ & $0.20 \pm 0.02$ & $0.14 \pm 0.02$ & $0.7 \pm 0.1$ & $3.21 \pm 0.07$ & $2.50 \pm 0.03$ \\
$\mathrm{~J} 025-33$ & 1192.47 & $0.38 \times 0.34$ & $<0.2$ & $<0.2$ & $<1.0$ & $0.76 \pm 0.05$ & $0.70 \pm 0.03$ \\
$\mathrm{~J} 029-36$ & 1145.16 & $0.34 \times 0.31$ & $0.33 \pm 0.02$ & $0.22 \pm 0.02$ & $1.8 \pm 0.2$ & $1.99 \pm 0.09$ & $1.17 \pm 0.03$ \\
\hline
\end{tabular}

Notes. (1) Source short name, (2) reference wavelength, (3) ALMA clean beam size, (4) Major axis FWHM of the continuum source deconvolved from beam, (5) Minor axis deconvolved FWHM, (6) Surface area of continuum emitting region, (7) IMFIT integrated fluxes, (8) IMFIT peak fluxes.

Table 6. SMG and quasar properties estimated from ALMA FIR/IR continuum flux densities and FIR sizes.

\begin{tabular}{|c|c|c|c|c|c|}
\hline Object & $\begin{array}{c}M_{\text {dust }} \\
\left(10^{8} \mathrm{M}_{\odot}\right) \\
(2)\end{array}$ & $\begin{array}{c}L_{\mathrm{FIR}} \\
\left(10^{12} \mathrm{~L}_{\odot}\right) \\
(3)\end{array}$ & $\begin{array}{c}\mathrm{SFR}_{\mathrm{IR}} \\
\left(\mathrm{M}_{\odot} \mathrm{yr}^{-1}\right) \\
(4)\end{array}$ & $\begin{array}{c}\text { Galaxy-averaged } \sum_{\mathrm{SFR}} \\
\left(\mathrm{M}_{\odot} \mathrm{yr}^{-1} \mathrm{kpc}^{-2}\right) \\
(5)\end{array}$ & $\begin{array}{c}\text { Peak } \sum_{\mathrm{SFR}} \\
\left(\mathrm{M}_{\odot} \mathrm{yr}^{-1} \mathrm{kpc}^{-2}\right) \\
(6)\end{array}$ \\
\hline LAB-01 & $5.0 \pm 1.0$ & $3.4 \pm 1.8$ & $663 \pm 359$ & $257 \pm 146$ & $265 \pm 143$ \\
\hline LAB-02 & $6.1 \pm 1.7$ & $3.7 \pm 3.0$ & $707 \pm 586$ & $184 \pm 155$ & $240 \pm 199$ \\
\hline LAB-03 & $12.0 \pm 3.6$ & $3.9 \pm 2.2$ & $760 \pm 437$ & $251 \pm 146$ & $264 \pm 152$ \\
\hline LAB-04 & $3.3 \pm 1.5$ & $1.1 \pm 0.7$ & $206 \pm 142$ & $98 \pm 86$ & $95 \pm 65$ \\
\hline LAB-05 & $8.9 \pm 1.3$ & $3.4 \pm 1.7$ & $659 \pm 323$ & $198 \pm 104$ & $204 \pm 100$ \\
\hline LAB-10 & $7.9 \pm 4.6$ & $<0.18^{*}$ & $<68^{*}$ & $<103^{*}$ & $<58^{*}$ \\
\hline LAB-11 & $1.9 \pm 0.5$ & $1.5 \pm 1.3$ & $301 \pm 258$ & $149 \pm 140$ & $390 \pm 333$ \\
\hline LAB-12 & $2.8 \pm 0.9$ & $0.9 \pm 0.5$ & $177 \pm 93$ & $175 \pm 155$ & $128 \pm 66$ \\
\hline J332-23 & $0.65 \pm 0.1$ & $1.0 \pm 0.4$ & $215 \pm 81$ & $<679^{*}$ & $48 \pm 18$ \\
\hline J158-14 & $4.1 \pm 0.4$ & $6.5 \pm 2.4$ & $1360 \pm 506$ & $943 \pm 472$ & $277 \pm 103$ \\
\hline J025-33 & $1.0 \pm 0.1$ & $1.6 \pm 0.6$ & $344 \pm 130$ & $>387^{\dagger}$ & $70 \pm 26$ \\
\hline J029-36 & $2.5 \pm 0.3$ & $4.0 \pm 1.5$ & $837 \pm 314$ & $219 \pm 98$ & $126 \pm 47$ \\
\hline
\end{tabular}

Notes. (1) Source short name, (2) Dust mass; these estimates are to be preferred over those quoted in Paper I, (3) FIR luminosity calculated using eq. 3 with rest wavelength limits between $42.5-122.5 \mu \mathrm{m}$, (4) IR star formation rate, (5) IR galaxy-averaged star formation rate surface density ( ${ }^{\dagger}$ signifies lower limit when maximum area of $1 \mathrm{kpc}^{2}$ for J025-33 is assumed), (6) IR peak star formation rate surface density (see text for details). In columns 3, 4, 5, 6 for LAB-10, ${ }^{*}$ signifies a $1 \sigma$ upper limit.

their different estimation method. Our peak SFR densities are similarly a factor of $2.2 \pm 0.19$ higher. One result is that the peak SFR density of ALESS 9.1 now appears above the Eddington limit. The known X-ray quasar ALESS 17.1 remains a factor of $\approx 7 \times$ below this limit.

All of the WHDF sub-mm sources lie below the Eddington limit and close to the ALESS sources in their SFR density, with the peak and integrated values generally being generallty similar within the errors for both the WHDF and $z>6$ sources. Here, the two known quasars LAB-05 and LAB-11 have similar SFR surface densities to the others. The slightly higher ALESS peak values relative to WHDF are probably due to their $\approx 30 \%$ higher resolution than the WHDF data.

For the four $z>6$ quasars, we see in Fig. 5 that the peak values are again slightly lower than those for ALESS and WHDF SMGs. Again these lower peak values might be explained by the $\approx 3 \times$ lower resolution in arcseconds, although given the $d_{\mathrm{A}}(z)$ relation, this is only $\approx 2 \times$ lower resolution measured in kiloparsecs.

Summarising, we have compared the integrated and peak $\sum_{\text {SFR }}$ values of our six WHDF SMGs with six known sub-mm-loud quasars. For comparison, we also include $\sum_{\mathrm{SFR}}$ measurements of the ALESS SMG sample, as presented in Fig. 6 of Hodge et al. (2019). We find that peak SFR density values generally increase with spatial resolution measured in kiloparsecs. Only one ALESS SMG exceeds the SFR Eddington limit but none of the others do, except for some highly resolved local U/LIRGs (see Fig. 6 of Hodge et al. 2019). The 5 WHDF SMGs show little difference with the 2 WHDF sub-mm quasars, the $4 z>6$ quasars and 5 out of 6 ALESS SMGs. The only further statement that can be made is that perhaps ALESS 9.1 with its super-Eddington SFR density might now join the X-ray source ALESS 17.1 in making at least 2 out of the 6 sources of Hodge et al. (2019) now identified as AGN. Although these statistics are still too poor to claim 1/3 of ALESS sources as a lower limit to the quasar fraction, we do note that ALESS 9.1 and 17.1 share similar low surface brightness galaxy bar and spiral arm features as the four other ALESS sources. So in interpreting these results, the morphological dissimilarity between ALESS and WHDF SMG samples will have to be explained by some more general sample characteristic e.g. FIR luminosity or $\mathrm{S} / \mathrm{N}$, rather than by sample bias towards one or other class of heating source.

\section{DISCUSSION: SMG AND QUASAR FIR PROPERTIES COMPARED}

In this work, we have presented high resolution ( $\left.0 .{ }^{\prime \prime} 1\right)$, band 7 , ALMA observations of eight $z \approx 2$ sub-mm sources originally detected by APEX LABOCA in the WHDF. Seven of these form a 


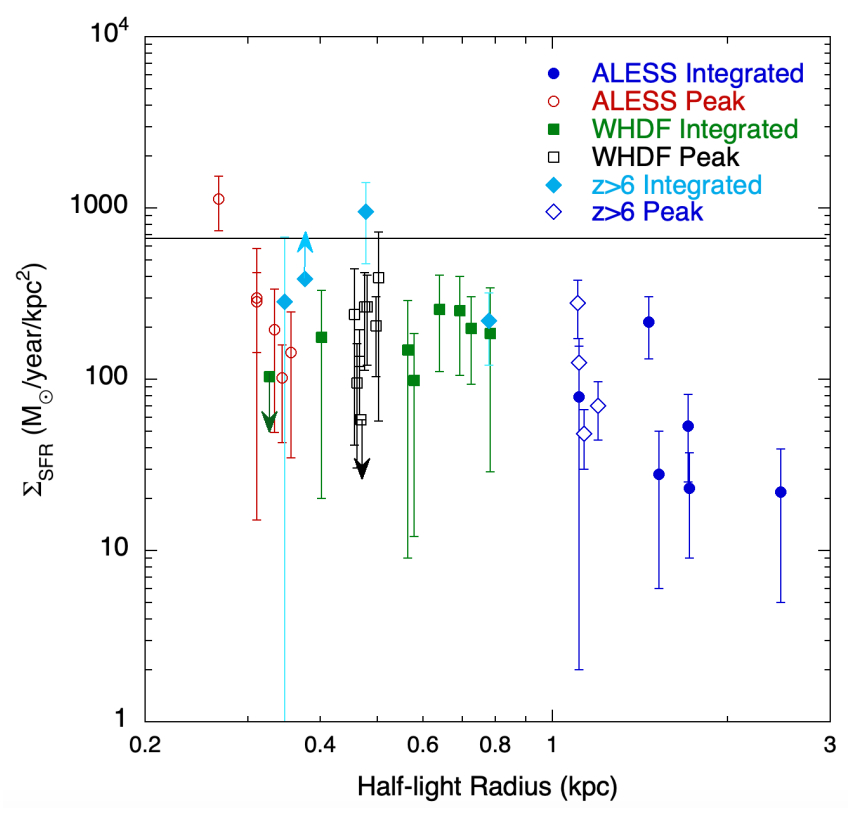

Figure 5. The integrated and peak star formation rate surface density $\left(\sum_{\mathrm{SFR}}\right)$ as a function of half-light radius for the ALESS SMGs of Hodge et al. (2019), compared to the WHDF SMGs and the $z \approx 6$ AGN presented in this work. The solid horizontal line indicates a lower bound for the Eddington limit for star formation as calculated by Hodge et al. (2019).

flux density limited sample in a central $7^{\prime} \times 7^{\prime}$ area of the WHDF. Two of these seven WHDF sources had been previously identified as X-ray absorbed quasars at $z=1.32$ and $z=2.12$. In addition, we presented our ALMA band 6 observations of four, $z>6$, quasars, initially detected in the VST ATLAS survey. We detected significant continuum dust emission in all four sources.

In Paper I, we looked for optical/NIR/MIR counterparts for the eight WHDF SMGs detected by LABOCA and ALMA. We found that where a counterpart was detected, most SMG SEDs were as well fitted by an obscured AGN SED as a star-forming SED. Photometric redshifts and dust absorptions were obtained. There were also several faint X-ray detections, bright enough to cause a further two sources to be classed as quasars making a minimum of 4/7 in the flux density limited WHDF sample. We used the original two WHDF quasars plus the four $z>6$ quasars as our comparison sample for the six WHDF SMGs.

All the WHDF sources were detected as resolved at $0 .{ }^{\prime \prime} 1$ resolution. Three out of four $z>6$ quasars were also more marginally resolved at $0 .{ }^{\prime \prime} 3$ angular resolution $(\approx 2 \mathrm{kpc}$ spatial) at $z \approx 6$ in the dust continuum emission. We find that all these sources are small in sub-mm extent i.e $\approx 1-2 \mathrm{kpc}$ with little difference between the six WHDF unidentified SMGs, the two WHDF quasars or the four $z>6$ quasars. They are also small relative to their host galaxies by a factor of $\approx 3$. In all cases, we find no features that can distinguish the six unidentified WHDF SMGs as being star-formation rather than AGN heated.

Our comparison of the ALMA FIR continuum extent of the WHDF SMGs with their resolved counterparts in $0 .{ }^{\prime \prime} 1$ resolution HST $i$-band imaging and $0 . " 9$ Calar Alto $H$-band imaging reveals that the ALMA FIR FWHM of these objects are generally only marginally smaller in the $i$-band by a factor of $1.6 \pm 0.36$ whereas they are consistently smaller than their $H$-band counterparts by a factor of $7.0 \pm 2.4$, or $4.8 \pm 0.36$ if LAB-04 is excluded on the grounds of its large error.

In this work, our primary goal was to identify the dominant fuelling mechanism behind the observed sub-mm emission of the SMGs. To this end, we used our ALMA observations of these sources to perform measurements of their sizes and star formation rates based on their continuum flux densities. We then used these measurements to calculate the star formation rate surface densities, $\sum_{\mathrm{SFR}}$, of the sub-mm emitting regions of these SMGs. We found none of these eight sources exceeded the Eddington limit as shown as a function of $\mathrm{R}_{e}$ and beam size in Fig. 5. In the case of our $z>6 \mathrm{SMGs}$ we found their integrated and peak $\sum_{\text {SFR }}$ values to bracket the SFR densities of the six known comparison quasars. Thus again there was little to distinguish SMGs from known quasars in our comparison sample.

In passing, we note that for five out of their sample of seven lensed, $1.5<z<3$, quasars, Stacey et al. (2021) measured small sub-mm sizes and correspondingly high star-formation rate densities, similar to those found here. Their remaining two quasars had $2-3 \times$ larger extent and thus lay well below the Eddington limit. These authors concluded that quasar sub-mm extents and implied star-formation rate densities are mostly similar to those for SMGs, in agreement with what we find here.

We also compared our results with those of the sample of 6 ALESS galaxies of Hodge et al. (2019). We found one ALESS SMG that exceeded the SFR Eddington limit so this sample now consisted of one probable quasar, one X-ray quasar and four unidentified SMGs. In terms of SFR density the 5 other ALESS SMGs spanned similar ranges to the WHDF SMGs and their quasar comparison sample. The major difference between the ALESS and WHDF samples was that the ALMA observations of the ALESS SMGs studied by Hodge et al. (2019) reveal clear signs of galactic sub-structure in these sources, whereas we observed no signs of low-surface brightness substructure in the ALMA observations of the WHDF SMGs. Within the ALESS sample, there also seemed little to distinguish the 2 quasars from the 4 others - all showed evidence of galaxy sub-structure. However, they all have more low surface brightness structure and larger $\mathrm{R}_{e}$ than the WHDF SMGs. Since Hodge et al. (2019) note that the only criterion used to select these sources for high resolution ALMA observations was their high luminosity, we conclude that this may drive this morphological difference, with perhaps a further contribution from the ALESS $\approx 2 \times$ longer ALMA Band 7 exposures.

We note that despite the larger size of the ALESS sub-structure relative to WHDF, their overall size is still small compared to the host galaxy sizes we are measuring. This means there is no problem in suggesting that these ALESS SMGs are AGN heated from the nucleus rather than from in situ star formation.

Both samples showed little difference between the AGN and SMG sub-samples in FIR luminosity and extent, relative size, dust masses, SFR surface densities and NIR + MIR luminosities. Some of these similarities are summarised in Fig. 6. Here, Fig. 6(a) compares the distribution of sub-mm effective radii, $\mathrm{R}_{\boldsymbol{e}}$, of the SMGs and QSOs from the $z<6$ QSO+WHDF and ALESS surveys. We first see that the $\mathrm{R}_{e}$ from our surveys clearly separate at $\mathrm{R}_{e} \approx 1 \mathrm{kpc}$ from those from ALESS, with the ALESS radii being systematically larger. We further note that within each of these sub-samples on either side of the vertical dashed line the SMG and QSO radii distributions appear very similar, with survey-survey systematic differences much larger. Next, Fig. 6 (b) now also includes 12 other WHDF quasars in a complete X-ray sample from Table 2 of Bielby et al. (2012). So Fig. 6 (b) shows the similarities in the distribution of the absolute magnitudes of the WHDF quasars and SMGs in the MIR [4.5] $\mu \mathrm{m}$ band (see Table A2 of Paper I). Here and throughout we have assumed $f_{\lambda} \propto \lambda^{-1}$ for $\lambda<4.5 \mu \mathrm{m}$ as is approximately the case for QSOs with absorption in the $0<A_{V}<2.5 \mathrm{mag}$ range estimated here. This can be verified by inspecting Figs. $5(\mathrm{a}, \mathrm{b})$ of Paper I. Assuming this spectral slope 

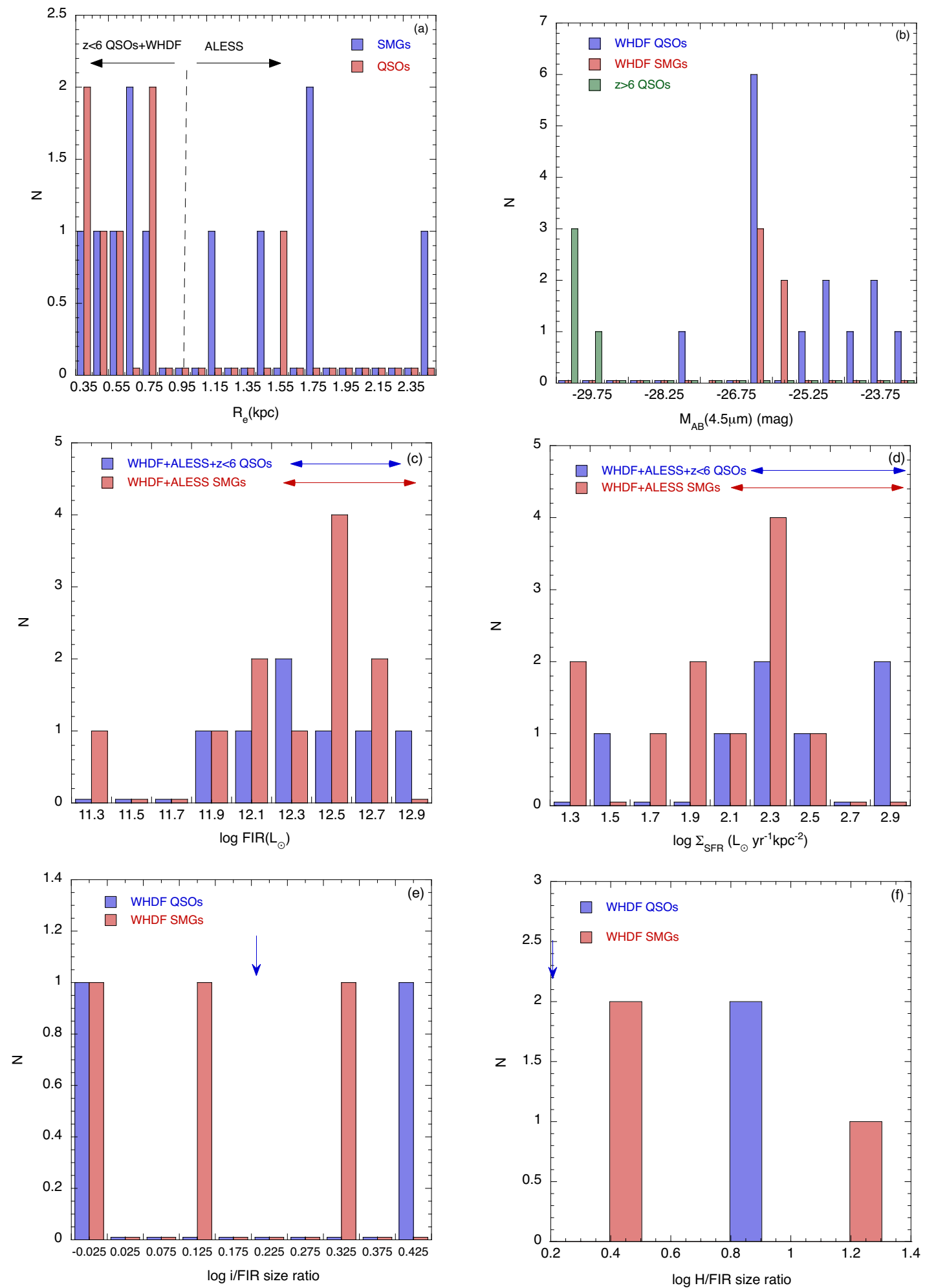

Figure 6. (a) Sub-mm effective radii, $\mathrm{R}_{e}$, compared for QSOs and SMGs with the $z<6+$ WHDF and ALESS results clearly separated on either side of the vertical dashed line, (b) Absolute magnitudes, $M_{\mathrm{AB}}(4.5 \mu \mathrm{m})$, compared for 14 WHDF X-ray QSOs (including LAB-05,-11 in $M_{\mathrm{AB}}(4.5 \mu \mathrm{m})=-26.25 \mathrm{mag}$ bin), 5 WHDF SMGs (Note that LAB-02 is undetected at $4.5 \mu \mathrm{m}$.) and $4 z>6$ quasars, (c) FIR luminosities compared for 2 WHDF (LAB-05, LAB-11) + 1 ALESS (ALESS 17.1) $+4 z>6$ quasars and $6 \mathrm{WHDF}+5$ ALESS SMGs; mean $\pm 1 \sigma=2 \sigma$ errors are shown, (d) SFR integrated surface densities compared for quasars and SMGs as in (c), (e) $i$-band/FIR continuum size ratios compared for WHDF quasars (LAB-05, LAB-011) and 3 SMGs (see Table 3 ). The arrow denotes the average $\left[\mathrm{C}_{\mathrm{II}}\right] /$ FIR continuum size ratio measured by Venemans et al. (2020) for a sample of $27 z \approx 6$ QSOs, (f) Same as (e) for $H$-band-FIR size ratio. 
then implies that the QSO K-correction is zero for the [4.5] $\mu \mathrm{m}$ band at all redshift and $A_{V}$ combinations considered here. The four $z>6$ quasars clearly are significantly brighter due to the dominant nuclear contribution from these extremely rare objects. The detection of the dust continuum in these high redshift quasars does, however, show that dust can still exist in quasar host galaxies under these conditions, close to a hyper-massive black hole.

Figs. 6 (c), (d) also confirm the similarities of the FIR luminosities and SFR densities of the WHDF+ALESS quasars and SMGs (see Table 6). Even the $z>6$ quasars are indistinguishable from the other quasars and SMGs in both these properties.

Figs. 6 (e), (f) show that the ratio of sizes of the FIR continua to the $i$-band and $H$-band host galaxy sizes are distributed similarly for the WHDF quasars and SMGs with the $H$-band ratios being larger (see also Table 3). The arrows show the average of the ratio of FIR continuum: $\left[\mathrm{C}_{\mathrm{II}}\right]$ extents as listed in Table 3 of Venemans et al. (2020) for the $27 z \approx 6$ quasars of Decarli et al. (2018). These show that the $\left[\mathrm{C}_{\mathrm{II}}\right]$ extents are generally $\approx 2 \times$ larger than the FIR dust continuum extents. They are also more in agreement with the $i$-band/FIR extent ratios than the larger $H$-band/FIR ratios. We note that several studies, e.g. Simpson et al. (2015); Elbaz et al. (2018); Gullberg et al. (2019); Franco et al. (2020); Gómez-Guijarro et al. (2021); Puglisi et al. (2021) argue that the small FIR size compared to the optical size is indicative of galaxies building their bulge. Although this scenario cannot be ruled out, it must be said that an $\approx 1 \mathrm{kpc}$ radius for an SMG powered by an AGN was predicted by many authors before the advent of ALMA So, for example, Granato \& Danese (1994); Andreani et al. (1999); Kuraszkiewicz et al. (2003); Hill \& Shanks (2011b) and also Siebenmorgen et al. (2015) predicted that any dust surrounding the central nucleus in high-z AGN must have outer radii of $\approx 1 \mathrm{kpc}$. On the basis of these a priori predictions and on the basis of the similarity of the distributions shown in Figs. 6 between SMGs and known quasars, we therefore suggest that AGN are as likely to power SMGs as star-formation.

\subsection{Do MIR colours and luminosities distinguish QSOs and SMGs?}

In Paper I, we noted that any model that implied that bright SMGs were AGN powered still had to explain the result in Fig. 1 of Hatziminaoglou et al. (2010) that sub-mm-loud, broad emission line, SDSS quasars show different Spitzer MIPS (Rieke et al. 2004) $S_{70 \mu \mathrm{m}} / S_{24 \mu \mathrm{m}}$ colours compared to fainter SMGs while showing similar Herschel SPIRE (Pilbratt et al. 2010; Griffin et al. 2010) $S_{350 \mu \mathrm{m}} / S_{250 \mu \mathrm{m}}$ colours at longer wavelengths. In Paper I, we then predicted that SMGs generally should show a dependence of $S_{70 \mu \mathrm{m}} / S_{24 \mu \mathrm{m}}$ colours on MIR luminosity ${ }^{9}$. Unfortunately, as noted in Sect. 2.4 of Paper I, the WHDF has no imaging coverage between $4.5-100 \mu \mathrm{m}$ so to investigate this issue further, we exploit the more extensive MIR coverage in the AS2UDS survey of Dudzevičiūte et al. (2020). In Fig. 7(a) we therefore plot $S_{100 \mu \mathrm{m}} / S_{24 \mu \mathrm{m}}$ versus absolute $4.5 \mu \mathrm{m}$ magnitudes for the 48/707 AS2UDS SMGs that have detections in these 3 bands so that this $S_{100 \mu \mathrm{m}} / S_{24 \mu \mathrm{m}}$ ratio may be used as a substitute for the $S_{70 \mu \mathrm{m}} / S_{24 \mu \mathrm{m}}$ ratio used by Hatziminaoglou et al. (2010). We see

\footnotetext{
9 Paper I suggested that WHDF SMGs appearing fainter in MIR flux than sub-mm quiet QSOs (see their Fig. 3) was evidence for such a luminosity dependence. However, Fig. 6(b) shows no such difference when absolute magnitude is considered, so no support for this hypothesis can be drawn from Fig. 3 of Paper I. In any case, the argument of Hatziminaoglou et al. (2010) applied to sub-mm-loud QSOs.
}

evidence for a correlation of the form required to explain the result of Hatziminaoglou et al. (2010). We further show that 6 candidate Chandra X-ray QSOs (Kocevski et al. 2018) which coincide with sub-mm sources in an AS2UDS sub-area, also tend to show systematically brighter $4.5 \mu \mathrm{m}$ luminosities and lower $S_{100 \mu \mathrm{m}} / S_{24 \mu \mathrm{m}}$ ratios.

We now perform a test to check if bright MIR luminosities correlate with lower $S_{100 \mu \mathrm{m}} / S_{24 \mu \mathrm{m}}$ because of the large incompleteness (48/707) of the sample shown in Fig. 7(a). To this end, we cut the sample down to the $68 \mathrm{SMGs}$ with a $24 \mu \mathrm{m}$ detection in the range $1<$ $z<1.7$. At these lower redshifts the $24 \mu \mathrm{m}$ sample is more complete with 43/68 having detections. Then, of these, 15 have $100 \mu \mathrm{m}$ detections and with the 28 non-detections now also providing more useful $S_{100 \mu \mathrm{m}} / S_{24 \mu \mathrm{m}}$ upper limits, the effective detection completeness increases from $7 \%$ to $63 \%$. We plot $S_{100 \mu \mathrm{m}} / S_{24 \mu \mathrm{m}}$ versus $M_{4.5 \mu \mathrm{m}}$ in Fig. 7(b). A maximum likelihood fit of the points shown give a linear fit of $\log _{10}\left(S_{100 \mu \mathrm{m}} / S_{24 \mu \mathrm{m}}\right)=0.16 \pm 0.03 \times M_{4.5}+5.45 \pm 0.3$ with the correlation significant at $\approx 3 \sigma$, supporting the reality of the apparent correlation seen in Fig. 7(a). Moreover, in Fig. 7(c) we similarly show $S_{24 \mu \mathrm{m}} / S_{8 \mu \mathrm{m}}$ versus $M_{4.5 \mu \mathrm{m}}$ for $267 / 707$ AS2UDS sources detected in all these 3 bands. Here the $24 \mu \mathrm{m}$ and $8 \mu \mathrm{m}$ data come from the $\approx 1 \mathrm{deg}^{2} \operatorname{SpUDS}$ survey (PI J. Dunlop). We find that this $38 \%$ completeness is at least enough to confirm the trend that brighter $4.5 \mu \mathrm{m}$ SMGs have lower ratios in these bands, similar to the above $S_{100 \mu \mathrm{m}} / S_{24 \mu \mathrm{m}}$ results. We also plot 22 candidate QSOs from the X-UDS Chandra X-ray $0.33 \mathrm{deg}^{2}$ survey of Kocevski et al. (2018) that are also listed in AS2UDS and have $[3.6 \mu \mathrm{m}]-[4.5 \mu \mathrm{m}]>0.5$ mag (Vega), an established MIR criterion for QSO selection (Stern et al. 2012). AS2UDS photometric redshifts are adopted for these. Also shown are 14 QSOs selected by K-excess in the UDS field by Smail et al. (2008), although only 3 are detected at $24 \mu \mathrm{m}$ and the other 11 represent $S_{24 \mu \mathrm{m}} / S_{8 \mu \mathrm{m}}$ upper limits in Fig. 7(c). 4 of these UDS QSOs overlap with the 22 candidate AS2UDS X-ray QSOs. We again see an apparent correlation between $4.5 \mu \mathrm{m}$ luminosity and now the $S_{24 \mu \mathrm{m}} / S_{8 \mu \mathrm{m}}$ ratio with both QSO samples populating the bright end of the distribution. We conclude first that the result in Fig. 7 (c) is in line with QSOs having lower $S_{70 \mu \mathrm{m}} / S_{24 \mu \mathrm{m}}$ ratios than fainter SMGs as found by Hatziminaoglou et al. (2010). Second, we conclude that the results in Figs. $7(\mathrm{a}, \mathrm{b}, \mathrm{c})$ all appear to suggest that sub-mm sources with brighter $4.5 \mu \mathrm{m}$ luminosities have lower $S_{100 \mu \mathrm{m}} / S_{24 \mu \mathrm{m}}$ and $S_{24 \mu \mathrm{m}} / S_{8 \mu \mathrm{m}}$ ratios. This result is naturally interpreted if more MIR luminous SMGs and sub-mm-loud QSOs have both hot and cold dust components, compared to less luminous sources that only show a cold dust component.

\subsection{Does the presence of low surface-brightness sub-structure depend on SMG FIR luminosity?}

Finally, we also try to explain the absence in all of the WHDF sample of low surface brightness sub-structure such as spiral arms seen in the sample of six ALESS SMGs of Hodge et al. (2019). Again we suggest that this could be a luminosity effect but here with the FIR sub-mm luminosity driving the different morphology seen in the higher luminosity SMG sample. However, some further contribution may arise from lower $\mathrm{S} / \mathrm{N}$ in the WHDF sub-mm sample compared to ALESS. This latter possibility can be further checked by significantly increasing the ALMA exposure time on the WHDF SMG sample. Either way, the ALESS result can still be explained by AGN nuclear heating, rather than in situ star-formation, heating the spiral arms since their extent is still small compared to the optical/NIR/[CII] extent of SMG host galaxies. 

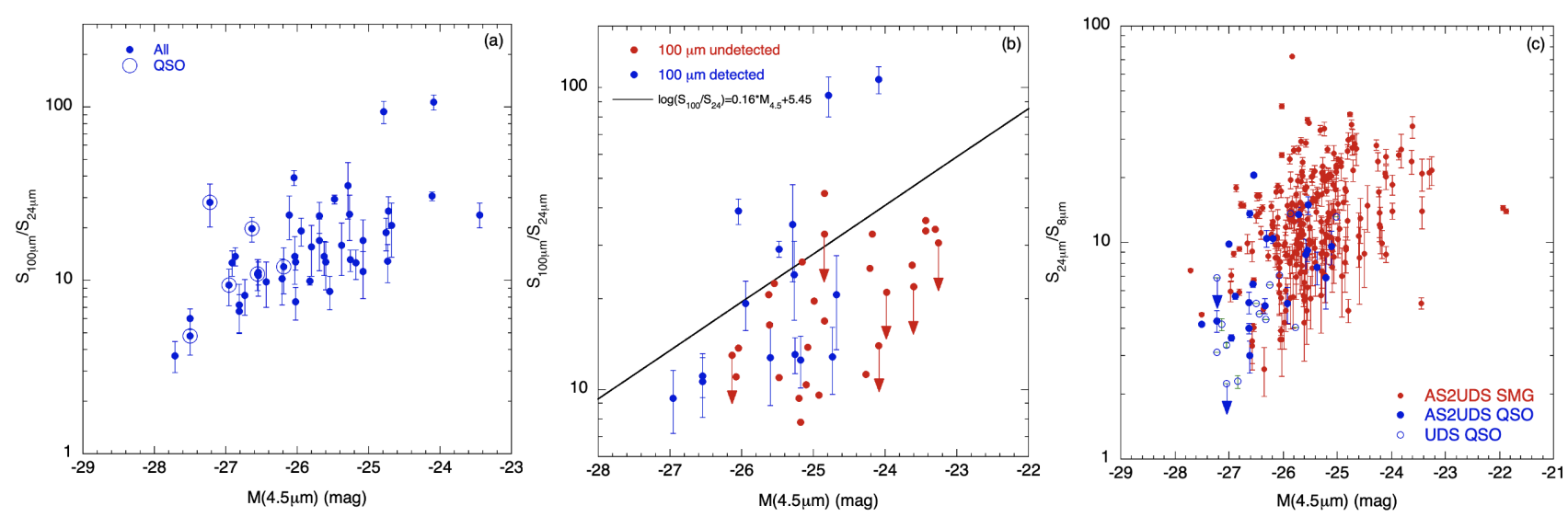

Figure 7. (a) $S_{100 \mu \mathrm{m}} / S_{24 \mu \mathrm{m}}$ flux density ratios plotted against $4.5 \mu \mathrm{m}$ absolute AB magnitudes for a sample of $48 / 707$ SMGs that have detections in all 3 bands from the AS2UDS survey of Dudzevičiūte et al. (2020). A correlation is seen as predicted in Paper I to explain why bright, sub-mm loud, SDSS QSOs have lower $S_{70 \mu \mathrm{m}} / S_{24 \mu \mathrm{m}}$ ratios than other SMGs (Hatziminaoglou et al. 2010), although selection effects may dominate this heavily cut sample. Also shown are 6 candidate Chandra X-ray QSOs (Kocevski et al. 2018) that are also AS2UDS detected (circled). These also tend to have brighter $4.5 \mu \mathrm{m}$ luminosities. (b) 43 AS2UDS SMGs with $1<z<1.7$ and with $24 \mu \mathrm{m}$ detections split into 15 with $100 \mu \mathrm{m}$ detections (blue) and 28 with only $100 \mu \mathrm{m}$ upper limits (red). The line is the maximum likelihood best fit. (c) $S_{24 \mu \mathrm{m}} / S_{8 \mu \mathrm{m}}$ ratios are also seen to correlate with $4.5 \mu \mathrm{m}$ absolute magnitudes (red filled circles) for 267 AS2UDS SMGs with detections in these 3 bands. 22 AS2UDS detected candidate X-ray QSOs (blue filled circles) from Kocevski et al. (2018) and 14 K-selected UDS QSOs (Smail et al. 2008) (blue open circles) generally show brighter $4.5 \mu \mathrm{m}$ luminosities and lower $S_{24 \mu \mathrm{m}} / S_{8 \mu \mathrm{m}}$ ratios.

\section{CONCLUSIONS}

We have observed eight WHDF sub-mm sources including two known absorbed X-ray quasars at $0 .{ }^{\prime \prime} 1$ resolution in ALMA and four VST ATLAS $z>6$ quasars at $0 . " 3$ resolution and resolved all but one $z>6$ quasar in the dust continuum. Our conclusions are as follows.

(i) As measured in the MIR (e.g. [4.5] $\mu \mathrm{m}$ ), the intrinsic luminosities of the WHDF SMGs and quasars in the sub-mm flux density limited sample are similar. They are also in the same range as mostly unabsorbed X-ray quasars in the complete WHDF X-ray sample that partly overlaps the sub-mm sample.

(ii) All the sub-mm sizes of the WHDF SMGs are compact $(\approx$ $1-2 \mathrm{kpc}$ ) with no difference in physical size compared to the two WHDF sub-mm-loud quasars or the four $z>6$ quasars.

(iii) All the sub-mm sizes of the WHDF SMGs are compact relative to the size of the host galaxy when detected in the optical or NIR. Again there is no difference in sub-mm - host relative extents between the six unidentified SMGs and the six quasars.

(iv) There is also little difference in either FIR luminosity or SFR surface density between the six WHDF SMGs and the six quasars. All lie below the SFR density 'Eddington limit' except for one $z>6$ quasar (and one ALESS SMG).

(v) There is a difference between our ALMA observations of the eight WHDF SMGs and the six ALESS SMGs observed by Hodge et al. (2019) in that the WHDF sources show no evidence of the low surface brightness spiral arms found in the ALESS sample. Deeper ALMA observations of the WHDF SMGs will show if this is due to the higher S/N of the ALESS sample or whether it is due to the ALESS SMG's higher sub-mm luminosity. Either way, the scale of the spiral arms remains so small that they are still consistent with AGN heating from the nucleus.

(vi) We find evidence in the AS2UDS survey (Dudzevičiūte et al. 2020) that sub-mm loud QSOs show lower $S_{100 \mu m} / S_{24 \mu \mathrm{m}}$ and $S_{24 \mu \mathrm{m}} / S_{8 \mu \mathrm{m}}$ flux density ratios than other SMGs, similar to the result of Hatziminaoglou et al. (2010). We also find preliminary evidence of a correlation between these flux density ratios and MIR $4.5 \mu \mathrm{m}$ luminosity. If this correlation is confirmed then it will suggest that more MIR luminous SMGs and sub-mm loud QSOs include hot as well as cold dust components. It would also remove the objection, based on the Hatziminaoglou et al. (2010) result, to the idea that most SMGs with $S_{870 \mu \mathrm{m}}>3 \mathrm{mJy}$ are AGN powered.

To these can be added the broad conclusion from Paper I that the unidentified WHDF SMGs with optical/NIR/MIR counterparts can be as well fitted by AGN SEDs as by star-forming galaxy SEDs. It should also be noted that the compact SMG sizes found by ALMA were a clear a priori prediction unique to the AGN powered SMG model. Indeed, the observed lack of dust heating at radii larger than $1-2 \mathrm{kpc}$ in galaxies that extend to $>8 \mathrm{kpc}$ could be taken as a strong signature for nuclear as opposed to in situ heating by local starformation. Our overall conclusion is therefore that there is no fundamental argument against AGN heating SMG dust rather than star formation. Indeed, the similarities between the AGN and SMG populations positively suggest that AGN heating may dominate at least in the brightest SMGs, allowing these dust-obscured quasars to help explain both the hard X-ray and FIR cosmic backgrounds.

\section{ACKNOWLEDGEMENTS}

We thank I.R. Smail for specific suggestions to improve Section 5.1. We also thank an anonymous referee for extensive suggestions that improved the quality of this paper throughout. BA acknowledges support from the Australian Research Council's Discovery Projects scheme (DP200101068). This paper makes use of the following ALMA data: ADS/JAO.ALMA-2016.1.01523.S and ADS/JAO.ALMA-2016.1.01510.S. ALMA is a partnership of ESO (representing its member states), NSF (USA) and NINS (Japan), together with NRC (Canada), MOST and ASIAA (Taiwan), and KASI (Republic of Korea), in cooperation with the Republic of Chile. The Joint ALMA Observatory is operated by ESO, AUI/NRAO and NAOJ. 


\section{DATA AVAILABILITY}

The catalogue data underlying this article are available in the article and Appendix A of Paper I. The imaging data underlying this article are publicly available in the ALMA archive.

\section{REFERENCES}

Alexander D. M., Bauer F. E., Chapman S. C., Smail I., Blain A. W., Brandt W. N., Ivison R. J., 2005, ApJ, 632, 736

Andreani P., Franceschini A., Granato G., 1999, MNRAS, 306, 161

Andrews B. H., Thompson T. A., 2011, ApJ, 727, 97

Barger A. J., Cowie L. L., Sanders D. B., Fulton E., Taniguchi Y., Sato Y., Kawara K., Okuda H., 1998, Nature, 394, 248

Baugh C. M., Lacey C. G., Frenk C. S., Granato G. L., Silva L., Bressan A., Benson A. J., Cole S., 2005, MNRAS, 356, 1191

Beelen A., Cox P., Benford D. J., Dowell C. D., Kovács A., Bertoldi F., Omont A., Carilli C. L., 2006, ApJ, 642, 694

Bielby R. M., Hill M. D., Metcalfe N., Shanks T., 2012, MNRAS, 419, 1315

Bizenberger P., McCaughrean M. J., Birk C., Thompson D., Storz C., 1998, in Fowler A. M., ed., Society of Photo-Optical Instrumentation Engineers (SPIE) Conference Series Vol. 3354, Infrared Astronomical Instrumentation. pp 825-832, doi:10.1117/12.317219

Blain A. W., Smail I., Ivison R. J., Kneib J. P., Frayer D. T., 2002, Phys. Rep., 369,111

Carnall A. C., et al., 2015, MNRAS, 451, L16

Casey C. M., Narayanan D., Cooray A., 2014, Phys. Rep., 541, 45

Chehade B., et al., 2018, MNRAS, 478, 1649

Comastri A., Setti G., Zamorani G., Hasinger G., 1995, A\&A, 296, 1

Cooke E. A., et al., 2018, ApJ, 861, 100

Cowie L. L., González-López J., Barger A. J., Bauer F. E., Hsu L. Y., Wang W. H., 2018, ApJ, 865, 106

Cowley W. I., Lacey C. G., Baugh C. M., Cole S., Frenk C. S., Lagos C. d. P., 2019, MNRAS, 487, 3082

Decarli R., et al., 2018, ApJ, 854, 97

Dey A., Graham J. R., Ivison R. J., Smail I., Wright G. S., Liu M. C., 1999, ApJ, 519, 610

Dudzevičiūte U., et al., 2020, MNRAS, 494, 3828

Elbaz D., et al., 2018, A\&A, 616, A110

Franco M., et al., 2018, A\&A, 620, A152

Franco M., et al., 2020, A\&A, 643, A53

Fujimoto S., Ouchi M., Shibuya T., Nagai H., 2017, ApJ, 850, 83

Geach J. E., et al., 2017, MNRAS, 465, 1789

Gómez-Guijarro C., et al., 2021, arXiv e-prints, p. arXiv:2106.13246

Granato G. L., Danese L., 1994, MNRAS, 268, 235

Griffin M. J., et al., 2010, A\&A, 518, L3

Gullberg B., et al., 2019, MNRAS, 490, 4956

Hatziminaoglou E., et al., 2010, A\&A, 518, L33

Heywood I., Bielby R. M., Hill M. D., Metcalfe N., Rawlings S., Shanks T., Smirnov O. M., 2013, MNRAS, 428, 935

Hill M. D., Shanks T., 2011a, MNRAS, 410, 762

Hill M. D., Shanks T., 2011b, MNRAS, 414, 1875

Hodge J. A., et al., 2013, ApJ, 768, 91

Hodge J. A., et al., 2016, ApJ, 833, 103

Hodge J. A., et al., 2019, ApJ, 876, 130

Holland W. S., et al., 1999, MNRAS, 303, 659

Hopkins P. F., Murray N., Quataert E., Thompson T. A., 2010, MNRAS, 401, L19

Hughes D. H., et al., 1998, Nature, 394, 241

Ikarashi S., et al., 2017, ApJ, 849, L36

Johnson S. P., et al., 2013, MNRAS, 431, 662

Kennicutt R. C., Evans N. J., 2012, ARA\&A, 50, 531

Kocevski D. D., et al., 2018, ApJS, 236, 48

Kuraszkiewicz J. K., et al., 2003, ApJ, 590, 128

Laird E. S., Nandra K., Pope A., Scott D., 2010, MNRAS, 401, 2763

Luo B., et al., 2017, ApJS, 228, 2

Magnelli B., et al., 2012, A\&A, 548, A22
McMullin J. P., Waters B., Schiebel D., Young W., Golap K., 2007, in Shaw R. A., Hill F., Bell D. J., eds, Astronomical Society of the Pacific Conference Series Vol. 376, Astronomical Data Analysis Software and Systems XVI. p. 127

Metcalfe N., Shanks T., Weilbacher P. M., McCracken H. J., Fong R., Thompson D., 2006, MNRAS, 370, 1257

Moran E. C., Lehnert M. D., Helfand D. J., 1999, ApJ, 526, 649

Pilbratt G. L., et al., 2010, A\&A, 518, L1

Polletta M., et al., 2007, ApJ, 663, 81

Puglisi A., et al., 2021, MNRAS,

Rieke G. H., et al., 2004, ApJS, 154, 25

Rujopakarn W., et al., 2016, ApJ, 833, 12

Rujopakarn W., et al., 2019, ApJ, 882, 107

Sanders D. B., Mirabel I. F., 1996, ARA\&A, 34, 749

Shanks T., et al., 2015, MNRAS, 451, 4238

Shanks T., Ansarinejad B., Bielby R. M., Heywood I., Metcalfe N., Wang L., 2021, MNRAS, 505, 1509

Siebenmorgen R., Heymann F., Efstathiou A., 2015, A\&A, 583, A120

Simpson J. M., et al., 2015, ApJ, 799, 81

Siringo G., et al., 2009, A\&A, 497, 945

Smail I., Ivison R. J., Blain A. W., 1997, ApJ, 490, L5

Smail I., Sharp R., Swinbank A. M., Akiyama M., Ueda Y., Foucaud S., Almaini O., Croom S., 2008, MNRAS, 389, 407

Stacey H. R., et al., 2021, MNRAS, 500, 3667

Stach S. M., et al., 2019, MNRAS, 487, 4648

Stern D., et al., 2012, ApJ, 753, 30

Swinbank A. M., et al., 2014, MNRAS, 438, 1267

Thompson T. A., Quataert E., Murray N., 2005, ApJ, 630, 167

Venemans B. P., et al., 2020, ApJ, 904, 130

Vernet J., et al., 2011, A\&A, 536, A105

Virtanen P., et al., 2020, scipy/scipy: SciPy 1.5.3, doi:10.5281/zenodo.4100507

Walter F., Riechers D., Cox P., Neri R., Carilli C., Bertoldi F., Weiss A., Maiolino R., 2009, Nature, 457, 699

Wang R., et al., 2013, ApJ, 773, 44

Willott C. J., Bergeron J., Omont A., 2017, ApJ, 850, 108

Worsley M. A., et al., 2005, MNRAS, 357, 1281

Zezas A. L., Georgantopoulos I., Ward M. J., 1998, MNRAS, 301, 915

This paper has been typeset from a $\mathrm{T}_{\mathrm{E}} \mathrm{X} / \mathrm{L} \mathrm{T} \mathrm{E} \mathrm{X}$ file prepared by the author. 


\section{University Library}

\section{- MINERVA \\ A gateway to Melbourne's research publications}

Minerva Access is the Institutional Repository of The University of Melbourne

\section{Author/s:}

Ansarinejad, B;Shanks, T;Bielby, RM;Metcalfe, N;Infante, L;Murphy, DNA;Rosario, DJ;Stach, SM

Title:

The nature of sub-millimetre galaxies II: an ALMA comparison of SMG dust heating mechanisms

Date:

2022-03

Citation:

Ansarinejad, B., Shanks, T., Bielby, R. M., Metcalfe, N., Infante, L., Murphy, D. N. A., Rosario, D. J. \& Stach, S. M. (2022). The nature of sub-millimetre galaxies II: an ALMA comparison of SMG dust heating mechanisms. Monthly Notices of the Royal Astronomical Society, 510 (4), pp.4976-4991. https://doi.org/10.1093/mnras/stac002.

Persistent Link:

http://hdl.handle.net/11343/297469 